\title{
Research Article \\ Exponential Admissibility and Dynamic Output Feedback Control of Switched Singular Systems with Interval Time-Varying Delay
}

\author{
Jinxing Lin and Chunxia Fan \\ College of Automation, Nanjing University of Posts and Telecommunications, Nanjing, \\ Jiangsu 210046, China \\ Correspondence should be addressed to Jinxing Lin, jxlin2004@126.com
}

Received 2 August 2010; Accepted 25 October 2010

Academic Editor: Cristian Toma

Copyright (C 2010 J. Lin and C. Fan. This is an open access article distributed under the Creative Commons Attribution License, which permits unrestricted use, distribution, and reproduction in any medium, provided the original work is properly cited.

\begin{abstract}
This paper is concerned with the problems of exponential admissibility and dynamic output feedback (DOF) control for a class of continuous-time switched singular systems with interval timevarying delay. A full-order, dynamic, synchronously switched DOF controller is considered. First, by using the average dwell time approach, a delay-range-dependent exponential admissibility criterion for the unforced switched singular time-delay system is established in terms of linear matrix inequalities (LMIs). Then, based on this criterion, a sufficient condition on the existence of a desired DOF controller, which guarantees that the closed-loop system is regular, impulse free and exponentially stable, is proposed by employing the LMI technique. Finally, some illustrative examples are given to show the effectiveness of the proposed approach.
\end{abstract}

\section{Introduction}

The past decades have witnessed an enormous interest in switched systems, due to their powerful ability in modeling of event-driven systems, logic-based systems, parameter- or structure-varying systems, and so forth; for details, see [1-4] and the references therein. Switched systems are a class of hybrid systems, which consist of a collection of continuousor discrete-time subsystems and a switching rule specifying the switching between them. When focusing on the classification problems in switched systems, it is commonly recognized that there exist three basic problems [1]: (i) finding conditions for stability under arbitrary switching; (ii) identifing the limited but useful class of stabilizing switching signals, and (iii) construct a stabilizing switching signal. Many effective methods have been presented to tackle these three basic problems such as the multiple Lyapunov function approach [5], the piecewise Lyapunov function approach [6], the switched Lyapunov function approach [7], 
the convex combination technique [8], and the dwell time or average dwell time scheme [912]. On the other hand, time-delay is very common in engineering systems and is frequently a source of instability and poor performance [13]. Therefore, control of switched time-delay systems has received more and more attention in the past few years; see [14-23] and the references therein.

As far as we know, singular systems (known also as descriptor, implicit or differential algebraic systems) also provide a natural framework for modeling of dynamic systems and describe a larger class of systems than the regular system models [24]. Switched singular systems have strong engineering background such as electrical networks [25], economic systems [26]. Recently, many results have been obtained in the literature for switched singular systems, such as stability and stabilization [27-30], reachability [31], $H_{\infty}$ control and filtering problems [32]. For switched singular time-delay (SSTD) systems, due to the coupling between the switching and the time-delay and because of the algebraic constraints in singular model, the behavior of such systems is much more complicated than that of regular switched time-delay systems or switched singular systems, and thus, to date, only a few results have been reported in the literature. In [33], the robust stability and $H_{\infty}$ control problems for discrete-time uncertain SSTD systems under arbitrary switching were discussed by using switched Lyapunov functions. In [34], a switching signal was constructed to guarantee the asymptotic stability of continuous-time SSTD systems. However, the aforementioned results are focused on the basic problem (i), see [33], and problem (iii), see [34], for SSTD systems. Problem (ii) is to identify stabilizing switching signals on the premise that all the individual subsystems are stable. Basically, we will find that stability is ensured if the switching is sufficiently slow [1], and it is well known that dwell time and average dwell time are two effective tools to define slow switching signals. In [9], it was shown that if all the individual subsystems are exponentially stable and that the dwell time of the switching signal is not smaller than a certain lower bound, then the switched systems is exponentially stable. This result was extended to both continuous-time switched linear time-delay systems [16] and discrete-time cases [17]. Unfortunately, so far, to the best of the authors' knowledge, the problem of solving the basic problem (ii) for SSTD systems via the dwell time or average dwell time scheme remains open and unsolved. On the other hand, the results in [33] are derived based on the state feedback controller. In fact, in many practical systems, state variables are not always available. In this case, the design of a controller that does not require the complete access to the state vector is preferable. An important example of such controller is the dynamic output feedback (DOF) controller. However, little attention has been paid to the DOF controller design problem for SSTD systems. This forms the motivation of this paper.

In this paper, we are concerned with the problems of exponential admissibility and DOF control for a class of continuous-time switched singular systems with interval timevarying delay. A full-order, dynamic, synchronously switched DOF controller is designed. First, by using the average dwell time approach and the piecewise Lyapunov function technique, a delay-range-dependent exponential admissibility criterion is derived in terms of LMIs, which guarantees the regularity, nonimpulsiveness, and exponential stability of the unforced system. A estimation of the convergence of the system is also explicitly given. Then, the corresponding solvability condition for the desired DOF controller is established by employing the LMI technique. Finally, some illustrative examples are given to show the effectiveness of the proposed approach.

Notation. Throughout this paper, $\mathbf{R}^{n}$ denotes the $n$-dimensional Euclidean space and $\mathbf{R}^{n \times n}$ is the set of all $n \times n$ real matrices. $P>0(P \geq 0)$ means that matrix $P$ is positive definite 
(semipositive definite). $\lambda_{\min }(P)\left(\lambda_{\max }(P)\right)$ denotes the minimum (maximum) eigenvalue of symmetric matrix $P . \mathcal{C}_{n, d}:=\mathcal{C}\left([-d, 0], \mathbf{R}^{n}\right)$ denotes the Banach space of continuous vector functions mapping the interval $[-d, 0]$ to $\mathbf{R}^{n}$. Let $x_{t} \in \mathcal{C}_{n, d}$ be defined by $x_{t}:=x(t+\theta)$, $\theta \in[-d, 0] .\|\cdot\|$ denotes the Euclidean norm of a vector and its induced norm of a matrix and $\left\|x_{t}\right\|_{d}=\sup _{-d \leq \theta \leq 0}\|x(t+\theta)\|$. The superscript " $T$ " represents matrix transposition, the symmetric terms in a matrix are denoted by " $*$ ". $\operatorname{diag}\{\cdots\}$ stands for a block-diagonal matrix and $\operatorname{Sym}\{A\}$ is the shorthand notation for $A+A^{T}$.

\section{Preliminaries and Problem Formulation}

Consider a class of SSTD systems of the form

$$
\begin{gathered}
E \dot{x}(t)=A_{\sigma(t)} x(t)+A_{d \sigma(t)} x(t-d(t))+B_{\sigma(t)} u(t), \\
y(t)=C_{\sigma(t)} x(t)+C_{d \sigma(t)} x(t-d(t)), \\
x(t)=\phi(t), \quad t \in\left[-d_{1}-d_{2}, 0\right],
\end{gathered}
$$

where $x(t) \in \mathbf{R}^{n}$ is the system state, $u(t) \in \mathbf{R}^{m}$ is the control input, and $y(t) \in \mathbf{R}^{p}$ is the measured output; $\sigma(t):[0,+\infty) \rightarrow \mathcal{O}=\{1,2, \ldots, N\}$ with integer $N>1$ is the switching signal; $E \in \mathbf{R}^{n \times n}$ is a singular matrix with rank $E=r \leq n$; for each possible value $\sigma(t)=i, i \in \mathcal{J}$, $A_{i}, A_{d i}, B_{i}, C_{i}$ and $C_{d i}$ are constant real matrices with appropriate dimensions; $\phi(t) \in \mathcal{C}_{n, d_{1}+d_{2}}$ is a compatible vector valued initial function; $d(t)$ is an interval time-varying delay satisfying

$$
d_{1} \leq d(t) \leq d_{1}+d_{2}, \quad \dot{d}(t) \leq \mu,
$$

where $d_{1} \geq 0, d_{2}>0$ and $0 \leq \mu<1$ are constants.

Remark 2.1. Model (2.1) can describe many practical time-delay systems (e.g., chemical engineering systems, lossless transmission lines, partial element equivalent circuit, etc.) with time-varying parameters or structures, which may be caused by random failures and repairs of the components, sudden environment changes, and varying of the operating point of a system $[13,35]$. In real application, the importance of the study of controller design problem for model (2.1) also arises from the extensive applications in networked control [36].

Since $\operatorname{rank} E=r \leq n$, there exist nonsingular transformation matrices $P, Q \in \mathbf{R}^{n \times n}$ such that $P E Q=\operatorname{diag}\left\{I_{r}, 0\right\}$. In this paper, without loss of generality, let

$$
E=\operatorname{diag}\left\{I_{r}, 0\right\}
$$

Corresponding to the switching signal $\sigma(t)$, we denote the switching sequence by $\mathcal{S}:=$ $\left\{\left(i_{0}, t_{0}\right), \ldots,\left(i_{k}, t_{k}\right) \mid i_{k} \in \mathcal{O}, k=0,1, \ldots\right\}$ with $t_{0}=0$, which means that the $i_{k}$ subsystem is activated when $t \in\left[t_{k}, t_{k+1}\right)$. To present the objective of this paper more precisely, the following definitions are introduced. 
Definition 2.2 (see $[16,37]$ ). For the switching signal $\sigma(t)$ and any delay $d(t)$ satisfying $(2.2)$, the unforced part of system (2.1)

$$
\begin{aligned}
& E \dot{x}(t)=A_{\sigma(t)} x(t)+A_{d \sigma(t)} x(t-d(t)), \\
& x_{t_{0}}(\theta)=x\left(t_{0}+\theta\right), \quad \theta \in\left[-d_{1}-d_{2}, 0\right]
\end{aligned}
$$

is said to be

(1) regular if $\operatorname{det}\left(s E-A_{i}\right)$ is not identically zero for each $\sigma(t)=i, i \in \mathcal{O}$,

(2) impulse free if $\operatorname{deg}\left(\operatorname{det}\left(s E-A_{i}\right)\right)=\operatorname{rank} E$ for each $\sigma(t)=i, i \in \mathcal{J}$,

(3) exponentially stable under the switching signal $\sigma(t)$ if the solution $x(t)$ of system (2.4) satisfies $\|x(t)\| \leq \iota e^{-\lambda\left(t-t_{0}\right)}\left\|x_{t_{0}}\right\|_{d_{1}+d_{2}}$, for all $t \geq t_{0}$, where $\lambda>0$ and $\iota>0$ are called the decay rate and decay coefficient, respectively;

(4) exponentially admissible if it is regular, impulse free and exponentially stable under the switching signal $\sigma(t)$.

Definition 2.3 (see [9]). For any $T_{2}>T_{1} \geq 0$, let $N_{\sigma}\left(T_{1}, T_{2}\right)$ denote the number of switching of $\sigma(t)$ over $\left(T_{1}, T_{2}\right)$. If $N_{\sigma}\left(T_{1}, T_{2}\right) \leq N_{0}+\left(T_{2}-T_{1}\right) / T_{a}$ holds for $T_{a}>0, N_{0} \geq 0$, then $T_{a}$ is called average dwell time. As commonly used in the literature, we choose $N_{0}=0$.

This paper considers the full-order DOF controller of the following form:

$$
\begin{gathered}
E_{c} \dot{x}_{c}(t)=A_{c \sigma(t)} x_{c}(t)+B_{c \sigma(t)} y(t), \\
u(t)=C_{c \sigma(t)} x_{c}(t)+D_{c \sigma(t)} y(t),
\end{gathered}
$$

where $x_{c}(t) \in \mathbf{R}^{n}$ is the controller state vector, and $E_{c}, A_{c i}, B_{c i}, C_{c i}$ and $D_{c i}, \sigma(t)=i, i \in \mathcal{O}$, are appropriately dimensioned constant matrices to be determined.

Then, the problem to be addressed in this paper can be formulated as follows: given the SSTD system (2.1), identify a class of switching signal $\sigma(t)$ and design a DOF controller of the form (2.5) such that the resultant closed-loop system is exponentially admissible under the switching signal $\sigma(t)$.

Before ending this section, we introduce the following lemma, which is essential for the development of our main results.

Lemma 2.4. For any constant matrix $Z \in R^{n \times n}, Z=Z^{T}>0$, positive scalar $\alpha$, and vector function $\dot{x}:[-\tau, \infty) \rightarrow R^{n}$ such that the following integration is well defined, then

$$
\frac{e^{\alpha \tau}-1}{\alpha} \int_{t-d(t)}^{t} e^{\alpha(s-t)} \dot{x}^{T}(s) E^{T} Z E \dot{x}(s) d s \geq\left(\int_{t-d(t)}^{t} E \dot{x}(s) d s\right)^{T} Z\left(\int_{t-d(t)}^{t} E \dot{x}(s) d s\right), \quad t \geq 0,
$$

where $0 \leq d(t) \leq \tau$. 
Proof. The proof is almost the same as Lemma 1 in [20]. Using Schur complement, we have

$$
\left[\begin{array}{cc}
e^{\alpha(s-t)} \dot{x}^{T}(s) E^{T} Z E \dot{x}(s) & \dot{x}^{T}(s) E^{T} \\
* & e^{\alpha(t-s)} Z^{-1}
\end{array}\right] \geq 0
$$

Integrating it from $t-d(t)$ to $t$, we get

$$
\left[\begin{array}{cc}
\int_{t-d(t)}^{t} e^{\alpha(s-t)} \dot{x}^{T}(s) E^{T} Z E \dot{x}(s) d s & \int_{t-d(t)}^{t} \dot{x}^{T}(s) E^{T} d s \\
* & \frac{\left(e^{\alpha \tau}-1\right) Z^{-1}}{\alpha}
\end{array}\right] \geq 0 .
$$

Using Schur complement again, we find that Lemma 2.4 holds.

\section{Main Results}

In this section, we first apply the average dwell time approach to investigate the exponential admissibility for SSTD system (2.4), and give the following result.

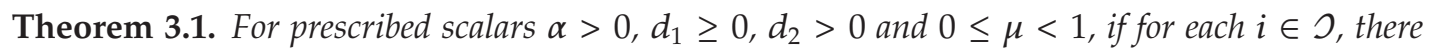
exist matrices $Q_{i l}>0, Z_{i l}>0, l=1,2$, and $P_{i}$ of the following form

$$
P_{i}=\left[\begin{array}{cc}
P_{i 11} & 0 \\
P_{i 21} & P_{i 22}
\end{array}\right]
$$

with $P_{i 11} \in \mathbf{R}^{r}, P_{i 11}>0$, and $P_{i 22}$ being invertible, such that

$$
\Phi_{i}=\left[\begin{array}{ccc}
\Phi_{i 11} & P_{i}^{T} A_{d i}+A_{i}^{T} U_{i} A_{d i} & c_{1} E^{T} Z_{i 1} E \\
* & \Phi_{i 22} & c_{2} E^{T} Z_{i 2} E \\
* & * & \Phi_{i 33}
\end{array}\right]<0
$$

where $\Phi_{i 11}=\operatorname{Sym}\left\{P_{i}^{T} A_{i}\right\}+\sum_{l=1}^{2} Q_{i l}+\alpha E^{T} P_{i}-c_{1} E^{T} Z_{i 1} E+A_{i}^{T} U_{i} A_{i}, \Phi_{i 22}=-(1-\mu) e^{-\alpha\left(d_{1}+d_{2}\right)} Q_{i 2}-$ $c_{2} E^{T} Z_{i 2} E+A_{d i}^{T} U_{i} A_{d i}, \Phi_{i 33}=-e^{-\alpha d_{1}} Q_{i 1}-c_{1} E^{T} Z_{i 1} E-c_{2} E^{T} Z_{i 2} E, c_{1}=\left(\alpha d_{1}\right) /\left(e^{\alpha d_{1}}-1\right), c_{2}=$ $\left(\alpha d_{2}\right) /\left(e^{\alpha d_{2}}-1\right)$ and $U_{i}=d_{1}^{2} Z_{i 1}+d_{2}^{2} e^{\alpha d_{1}} Z_{i 2}$. Then, system (2.4) with $d(t)$ satisfying (2.2) is exponentially admissible for any switching sequence $\mathcal{S}$ with average dwell time $T_{a} \geq T_{a}^{*}=(\ln \beta) / \alpha$, where $\beta \geq 1$ satisfies

$$
P_{i 11} \leq \beta P_{j 11}, \quad Q_{i l} \leq \beta Q_{j l}, \quad Z_{i l} \leq \beta Z_{j l}, \quad l=1,2, \quad \forall i, j \in \supset .
$$

Moreover, an estimate on the exponential decay rate is $\lambda=(1 / 2)\left(\alpha-(\ln \beta) / T_{a}\right)$. 
Proof. The proof is divided into three parts: (i) to show the regularity and nonimpulsiveness; (ii) to show the exponential stability of the differential subsystem; and (iii) to show the exponential stability of the algebraic subsystem.

Part (i) regularity and nonimpulsiveness. According to (2.3), for each $i \in \mathcal{O}$, denote

$$
A_{i}=\left[\begin{array}{ll}
A_{i 11} & A_{i 12} \\
A_{i 21} & A_{i 22}
\end{array}\right], \quad Z_{i 1}=\left[\begin{array}{ll}
Z_{i 111} & Z_{i 112} \\
Z_{i 112}^{T} & Z_{i 122}
\end{array}\right]
$$

where $A_{i 11} \in \mathbf{R}^{r}$ and $Z_{i 111} \in \mathbf{R}^{r}$. From (3.2), it is easy to see that $\Phi_{i 11}<0, i \in$ O. Noting $Q_{i l}>0$ and $Z_{i l}>0, l=1,2$, we get $\operatorname{Sym}\left\{P_{i}^{T} A_{i}\right\}+\alpha E^{T} P_{i}-c_{1} E^{T} Z_{i 1} E<0$. Substituting $P_{i}, A_{i}$, $Z_{i 1}$ and $E$ given as (3.1), (3.4) and (2.3) into this inequality and using Schur complement, we have $\operatorname{Sym}\left\{A_{i 22}^{T} P_{i 22}\right\}<0$, which implies that $A_{i 22}, i \in \mathcal{O}$, is nonsingular. Then by [24] and Definition 2.2, system (2.4) is regular and impulse free.

Part (ii) exponential stability of the differential subsystem. Define the piecewise Lyapunov functional candidate for system (2.4) as follows

$$
\begin{aligned}
V\left(x_{t}\right)= & V_{\sigma(t)}\left(x_{t}\right) \\
= & x^{T}(t) E^{T} P_{\sigma(t)} x(t)+\int_{t-d_{1}}^{t} e^{\alpha(s-t)} x^{T}(s) Q_{\sigma(t) 1} x(s) d s \\
& +\int_{t-d(t)}^{t} e^{\alpha(s-t)} x^{T}(s) Q_{\sigma(t) 2} x(s) d s \\
& +d_{1} \int_{-d_{1}}^{0} \int_{t+\theta}^{t} e^{\alpha(s-t)}(E \dot{x}(s))^{T} Z_{\sigma(t) 1}(E \dot{x}(s)) d s d \theta \\
& +d_{2} \int_{-d_{1}-d_{2}}^{-d_{1}} \int_{t+\theta}^{t} e^{\alpha\left(s-t+d_{1}\right)}(E \dot{x}(s))^{T} Z_{\sigma(t) 2}(E \dot{x}(s)) d s d \theta .
\end{aligned}
$$

As mentioned earlier, the $i_{k}$ th subsystem is activated when $t \in\left[t_{k}, t_{k+1}\right)$. Then, along the solution of system (2.4) under the switching sequence $\mathcal{S}$, for $t \in\left[t_{k}, t_{k+1}\right)$, we have

$$
\begin{aligned}
\dot{V}_{i_{k}}\left(x_{t}\right)+\alpha V_{i_{k}}\left(x_{t}\right) \leq & 2 x^{T}(t) P_{i_{k}}^{T} E \dot{x}(t)+x^{T}(t) Q_{i_{k}} x(t)-e^{-\alpha d_{1}} x^{T}\left(t-d_{1}\right) Q_{i_{k}} x\left(t-d_{1}\right) \\
& +x^{T}(t) Q_{i_{k} 2} x(t)-(1-\mu) e^{-\alpha\left(d_{1}+d_{2}\right)} x^{T}(t-d(t)) Q_{i_{k} 2} x(t-d(t)) \\
& +(E \dot{x}(t))^{T}\left(d_{1}^{2} Z_{i_{k} 1}+d_{2}^{2} e^{\alpha d_{1}} Z_{i_{k} 2}\right)(E \dot{x}(t))+\alpha x^{T}(t) E^{T} P_{i_{k}} x(t) \\
& -d_{1} \int_{t-d_{1}}^{t} e^{\alpha(s-t)}(E \dot{x}(s))^{T} Z_{i_{k} 1}(E \dot{x}(s)) d s \\
& -d_{2} \int_{t-d(t)}^{t-d_{1}} e^{\alpha\left(s-t+d_{1}\right)}(E \dot{x}(s))^{T} Z_{i_{k} 2}(E \dot{x}(s)) d s .
\end{aligned}
$$


By replacing $E \dot{x}(t)$ with $A_{i_{k}} x(t)+A_{d i_{k}} x(t-d(t))$ and using Lemma 2.4 and Schur complement, LMI (3.2) yields

$$
\dot{V}_{i_{k}}\left(x_{t}\right)+\alpha V_{i_{k}}\left(x_{t}\right)<0 .
$$

Integrating (3.7) from $t_{k}$ to $t_{k+1}$ gives

$$
V_{\sigma(t)}\left(x_{t}\right)=V_{i_{k}}\left(x_{t}\right) \leq e^{-\alpha\left(t-t_{k}\right)} V_{i_{k}}\left(x_{t_{k}}\right), \quad t \in\left[t_{k}, t_{k+1}\right) .
$$

Let $x(t)=\left[\begin{array}{l}x_{1}(t) \\ x_{2}(t)\end{array}\right]$, where $x_{1}(t) \in \mathbf{R}^{r}$ and $x_{2}(t) \in \mathbf{R}^{n-r}$. From (2.3) and (3.1), it can be seen that for each $i, i \in \mathcal{O}, x^{T}(t) E^{T} P_{i} x(t)=x_{1}^{T}(t) P_{i 11} x_{1}(t)$. Noting this, and using (3.3) and (3.5), at switching instant $t_{k}$, we have

$$
V_{i_{k}}\left(x_{t_{k}}\right) \leq \beta V_{\sigma\left(t_{k}^{-}\right)}\left(x_{t_{k}^{-}}\right)=\beta V_{i_{k-1}}\left(x_{t_{k}^{-}}\right), \quad k=1,2, \ldots
$$

where $t_{k}^{-}$denotes the left limitation of $t_{k}$. Therefore, it follows from (3.8), (3.9) and the relation $k=N_{\sigma}\left(t_{0}, t\right) \leq\left(t-t_{0}\right) / T_{a}$ that

$$
\begin{aligned}
V_{\sigma(t)}\left(x_{t}\right) & \leq e^{-\alpha\left(t-t_{k}\right)} \beta V_{\sigma\left(t_{k}^{-}\right)}\left(x_{t_{k}^{-}}\right) \leq \cdots \leq e^{-\alpha\left(t-t_{0}\right)} \beta^{k} V_{\sigma\left(t_{0}\right)}\left(t_{0}\right) \\
& \leq e^{-\left(\alpha-(\ln \beta) / T_{a}\right)\left(t-t_{0}\right)} V_{\sigma\left(t_{0}\right)}\left(x_{t_{0}}\right)
\end{aligned}
$$

According to (3.5) and (3.10), we obtain

$$
\lambda_{1}\left\|x_{1}(t)\right\|^{2} \leq V_{\sigma(t)}\left(x_{t}\right), \quad V_{\sigma\left(t_{0}\right)}\left(x_{t_{0}}\right) \leq \lambda_{2}\left\|x_{t_{0}}\right\|_{d_{1}+d_{2}}^{2}
$$

where $\lambda_{1}=\min _{\forall i \in O} \lambda_{\min }\left(P_{i 11}\right)$, and $\lambda_{2}=\max _{\forall i \in \supset} \lambda_{\max }\left(P_{i 11}\right)+(1 / \alpha)\left(1-e^{-\alpha d_{1}}\right) \max _{\forall i \in O} \lambda_{\max }\left(Q_{i 1}\right)+$ $(1 / \alpha)\left(1-e^{-\alpha\left(d_{1}+d_{2}\right)}\right) \max _{\forall i \in \rho} \lambda_{\max }\left(Q_{i 2}\right)+\left(d_{1} / \alpha^{2}\right)\left(\alpha d_{1}-1+e^{-\alpha d_{1}}\right) \max _{\forall i \in \mathcal{S}}\left(2 \lambda_{\max }\left(Z_{i 1}\right)\left(\left\|A_{i}\right\|+\right.\right.$ $\left.\left.\left\|A_{d i}\right\|\right)\right)+\left(1 / \alpha^{2}\right)\left(-d_{2}+\alpha d_{2}^{2} e^{\alpha d_{1}}+d_{2} e^{-\alpha d_{2}}\right) \max _{\forall i \in \mathcal{O}}\left(2 \lambda_{\max }\left(Z_{i 2}\right)\left(\left\|A_{i}\right\|+\left\|A_{d i}\right\|\right)\right)$. Then, combining (3.10) with (3.11) yields

$$
\left\|x_{1}(t)\right\| \leq \sqrt{\frac{\lambda_{2}}{\lambda_{1}}} e^{-(1 / 2)\left(\alpha-(\ln \beta) / T_{a}\right)\left(t-t_{0}\right)}\left\|x_{t_{0}}\right\|_{d_{1}+d_{2}} .
$$

Part (iii) exponential stability of the algebraic subsystem. Since $A_{i 22}, i \in \mathcal{O}$, is nonsingular, set $G_{i}=\left[\begin{array}{cc}I_{r} & -A_{i 22} A_{i 22}^{-1} \\ 0 & A_{i 22}^{-1}\end{array}\right]$ and $H=\left[\begin{array}{cc}I_{r} & 0 \\ 0 & I_{n-r}\end{array}\right]$. Then, it is easy to get

$$
\widehat{E}:=G_{i} E H=\left[\begin{array}{cc}
I_{r} & 0 \\
0 & 0
\end{array}\right], \quad \widehat{A}_{i}:=G_{i} A_{i} H=\left[\begin{array}{cc}
\widehat{A}_{i 11} & 0 \\
\widehat{A}_{i 21} & I_{n-r}
\end{array}\right], \quad \widehat{P}_{i}:=G_{i}^{-T} P_{i} H=\left[\begin{array}{cc}
\widehat{P}_{i 11} & 0 \\
\widehat{P}_{i 21} & \widehat{P}_{i 22}
\end{array}\right],
$$


where $\widehat{A}_{i 11}=A_{i 11}-A_{i 12} A_{i 22}^{-1} A_{i 21}, \widehat{A}_{i 21}=A_{i 22}^{-1} A_{i 21}, \widehat{P}_{i 11}=P_{i 11}, \widehat{P}_{i 21}=A_{i 12}^{T} P_{i 11}+A_{i 22}^{T} P_{i 21}$, and $\widehat{P}_{i 22}=A_{i 22}^{T} P_{i 22}$. According to (3.13), denote

$$
\begin{gathered}
\widehat{A}_{d i}:=G_{i} A_{d i} H=\left[\begin{array}{cc}
\widehat{A}_{d i 11} & \widehat{A}_{d i 12} \\
\widehat{A}_{d i 21} & \widehat{A}_{d i 22}
\end{array}\right], \quad \widehat{Q}_{i l}:=H^{T} Q_{i l} H=\left[\begin{array}{ll}
\widehat{Q}_{i l 11} & \widehat{Q}_{i l 12} \\
\widehat{Q}_{i l 21} & \widehat{Q}_{i l 22}
\end{array}\right], \\
\hat{Z}_{i l}:=G_{i}^{-T} Z_{i l} G_{i}^{-1}=\left[\begin{array}{ll}
\widehat{Z}_{i l 11} & \widehat{Z}_{i l 12} \\
\widehat{Z}_{i l 21} & \widehat{Z}_{i l 22}
\end{array}\right], \quad l=1,2 .
\end{gathered}
$$

Let

$$
\xi(t)=\left[\begin{array}{l}
\xi_{1}(t) \\
\xi_{2}(t)
\end{array}\right]:=H^{-1} x(t)=x(t),
$$

where $\xi_{1}(t) \in \mathbf{R}^{r}$ and $\xi_{2}(t) \in \mathbf{R}^{n-r}$. Then, for any fixed $\sigma(t)=i, i \in \mathcal{O}$, system (2.4) is restricted system equivalent (r.s.e.) to

$$
\begin{aligned}
& \dot{\xi}_{1}(t)=\widehat{A}_{i 11} \xi_{1}(t)+\widehat{A}_{d i 11} \xi_{1}(t-d(t))+\widehat{A}_{d i 12} \xi_{2}(t-d(t)) \\
& -\xi_{2}(t)=\widehat{A}_{i 21} \xi_{1}(t)+\widehat{A}_{d i 21} \xi_{1}(t-d(t))+\widehat{A}_{d i 22} \xi_{2}(t-d(t)) .
\end{aligned}
$$

By (3.2) and Schur complement, we have $\left[\begin{array}{cc}\Phi_{i 11}^{\prime} & P_{i}^{T} A_{d i} \\ * & \Phi_{i 22}^{\prime}\end{array}\right]<0$, where $\Phi_{i 11}^{\prime}=\operatorname{Sym}\left\{P_{i}^{T} A_{i}\right\}+\sum_{l=1}^{2} Q_{i l}+$ $\alpha E^{T} P_{i}-c_{1} E^{T} Z_{i 1} E$ and $\Phi_{i 22}^{\prime}=-(1-\mu) e^{-\alpha\left(d_{1}+d_{2}\right)} Q_{i 2}-c_{2} E^{T} Z_{i 2} E$. Pre- and postmultiplying this inequality by $\operatorname{diag}\left\{H^{T}, H^{T}\right\}$ and $\operatorname{diag}\{H, H\}$, respectively, noting the expressions in (3.13) and (3.14), and using Schur complement, we have

$$
\left[\begin{array}{cc}
\operatorname{Sym}\left\{\widehat{P}_{i 22}^{T}\right\}+\sum_{l=1}^{2} \widehat{Q}_{i l 22} & \widehat{P}_{i 22}^{T} \widehat{A}_{d i 22} \\
* & -(1-\mu) e^{-\alpha\left(d_{1}+d_{2}\right)} \widehat{Q}_{i 222}
\end{array}\right]<0 .
$$

Pre- and postmultiplying this inequality by $\left[-\widehat{A}_{d i 22}^{T} I\right]$ and its transpose, respectively, and noting $\widehat{Q}_{i 122}>0$ and $0 \leq \mu<1$, we obtain $\widehat{A}_{d i 22}^{T} \widehat{Q}_{i 222} \widehat{A}_{d i 22}-e^{-\alpha\left(d_{1}+d_{2}\right)} \widehat{Q}_{i 222}<0$. Then, according to Lemma 7 in [38], we can deduce that there exist constants $\hbar_{i}>1$ and $\eta_{i}>0$ such that

$$
\left\|\left(e^{(1 / 2) \alpha\left(d_{1}+d_{2}\right)} \widehat{A}_{d i 22}\right)^{l}\right\| \leq \hbar_{i} e^{-\eta_{i} l}, \quad l=0,1, \ldots, \forall i \in \supset
$$

Define

$$
\begin{gathered}
t^{0}=t, \quad t^{j}=t^{j-1}-d\left(t^{j-1}\right), \quad j=1,2, \ldots, \\
\left\|\widehat{A}_{21}\right\|=\max _{\forall i \in J}\left\|\widehat{A}_{i 21}\right\|, \quad\left\|\widehat{A}_{d 21}\right\|=\max _{\forall i \in J}\left\|\widehat{A}_{d i 21}\right\|, \quad\left\|\widehat{A}_{d 22}\right\|=\max _{\forall i \in \supset}\left\|\widehat{A}_{d i 22}\right\|, \quad \forall i \in \supset .
\end{gathered}
$$


As mentioned earlier, under the switching sequence $\mathcal{S}$, for $t \in\left[t_{k}, t_{k+1}\right)$, the $i_{k}$ th subsystem is activated. Then, from (3.17) and (3.20), we have

$$
\xi_{2}(t)-\widehat{A}_{i_{k} 21} \xi_{1}\left(t^{0}\right)-\widehat{A}_{d i_{k} 21} \xi_{1}\left(t^{1}\right)-\widehat{A}_{d i_{k} 22} \xi_{2}\left(t^{1}\right)
$$

Similarly, it can be obtained that $\xi_{2}\left(t^{1}\right)=-\widehat{A}_{i_{k} 21} \xi_{1}\left(t^{1}\right)-\widehat{A}_{d i_{k} 21} \xi_{1}\left(t^{2}\right)-\widehat{A}_{d i_{k} 22 \xi_{2}}\left(t^{2}\right)$. Substituting this into (3.22), we get $\xi_{2}(t)=\left(-\widehat{A}_{d i_{k} 22}\right)^{2} \xi_{2}\left(t^{2}\right)-\sum_{j=0}^{1}\left(-\widehat{A}_{d i_{k} 22}\right)^{j}\left(\widehat{A}_{i_{k} 21} \xi_{1}\left(t^{j}\right)+\widehat{A}_{d i_{k} 21} \xi_{1}\left(t^{j+1}\right)\right)$. Continuing in the same manner and noting that $t^{j}<t^{j-1}$, then there exists a finite positive integer $T_{i_{k}}$ such that

$$
\xi_{2}(t)=\left(-\widehat{A}_{d i_{k} 22}\right)^{T_{i_{k}}} \xi_{2}\left(t^{T_{i_{k}}}\right)-\sum_{j_{i_{k}}=0}^{T_{i_{k}}-1}\left(-\widehat{A}_{d i_{k} 22}\right)^{j_{i_{k}}}\left(\widehat{A}_{i_{k} 21} \xi_{1}\left(t^{j_{i_{k}}}\right)+\widehat{A}_{d i_{k} 21} \xi_{1}\left(t^{j_{i_{k}}+1}\right)\right)
$$

where $t^{T_{i_{k}}} \in\left(t_{k-1}, t_{k}\right]$ and $t^{T_{i_{k}}} \rightarrow t_{k}$. When $t \in\left[t_{k-1}, t_{k}\right)$, the $i_{k-1}$ th subsystem is activated. Then, following a similar procedure as the above, there exists a finite positive integer $T_{i_{k-1}}$ such that

$$
\begin{aligned}
\xi_{2}\left(t^{T_{i_{k}}}\right)= & \left(-\widehat{A}_{d i_{k-1} 22}\right)^{T_{i_{k-1}}} \xi_{2}\left(t^{T_{i_{k}}+T_{i_{k-1}}}\right) \\
& -\sum_{j_{i_{k-1}}=T_{i_{k}}}^{T_{i_{k}}+T_{i_{k-1}}-1}\left(-\widehat{A}_{d i_{k-1} 22}\right)^{j_{i_{k-1}}-T_{i_{k}}}\left(\widehat{A}_{i_{k-1} 21} \xi_{1}\left(t^{j_{i_{k-1}}}\right)+\widehat{A}_{d i_{k-1} 21} \xi_{1}\left(t^{j_{i_{k-1}}+1}\right)\right),
\end{aligned}
$$

where $t^{T_{i_{k}}+T_{i_{k-1}}} \in\left(t_{k-2}, t_{k-1}\right]$ and $t^{T_{i_{k}}+T_{i_{k-1}}} \rightarrow t_{k-1}$. After $k$-times iterative manipulations, $t$ belongs to $\left[t_{0}, t_{1}\right)$, and there exists a finite positive integer $T_{i_{0}}$ such that

$$
\begin{aligned}
\xi_{2}\left(t^{T_{i_{k}}+\cdots+T_{i_{1}}}\right) & =\left(-\widehat{A}_{d i_{0} 22}\right)^{T_{i_{0}}} \xi_{2}\left(t^{T_{i_{k}}+\cdots+T_{i_{0}}}\right) \\
& -\sum_{j_{i_{0}}=T_{i_{k}}+\cdots+T_{i_{1}}}^{T_{i_{k}}+\cdots+T_{i_{0}}-1}\left(-\widehat{A}_{d i_{0} 22}\right)^{j_{i_{0}}-T_{i_{k}}-\cdots-T_{i_{1}}}\left(\widehat{A}_{i_{0} 21} \xi_{1}\left(t^{j_{i_{0}}}\right)+\widehat{A}_{d i_{0} 21} \xi_{1}\left(t^{j_{i_{0}}+1}\right)\right),
\end{aligned}
$$

where $t^{T_{i_{k}}+\cdots+T_{i_{0}}} \in\left(-d_{1}-d_{2}, t_{0}\right]$ and $t^{T_{i_{k}}+\cdots+T_{i_{0}}} \rightarrow t_{0}$. By a simple induction, we have

$$
\begin{aligned}
\xi_{2}(t)= & {\left[\prod_{j=0}^{k}\left(-\widehat{A}_{d i_{j} 22}\right)^{T_{i_{j}}}\right] \xi_{2}\left(t^{T_{i_{k}}+\cdots+T_{i_{1}}+T_{i_{0}}}\right)-\sum_{j_{i_{k}}=0}^{T_{i_{k}}-1}\left(-\widehat{A}_{d i_{k} 22}\right)^{j_{i_{k}}}\left(\widehat{A}_{i_{k} 21} \xi_{1}\left(t^{j_{i_{k}}}\right)+\widehat{A}_{d i_{k} 21} \xi_{1}\left(t^{j_{i_{k}}+1}\right)\right) } \\
& -\sum_{p=1}^{k}\left\{\left[\prod_{q=p}^{k}\left(-\widehat{A}_{d i_{q} 22}\right)^{T_{i_{q}}}\right]_{j_{i_{p-1}}=T_{i_{k}}+\cdots+T_{i_{p}}}^{T_{i_{k}+\cdots+T_{i_{p-1}}-1}}\left(\varphi_{1}(t)+\varphi_{2}(t)\right)\right\},
\end{aligned}
$$


where

$$
\begin{gathered}
\varphi_{1}(t)=\left(-\widehat{A}_{d i_{p-1} 22}\right)^{j_{i_{p-1}}-T_{i_{k}}-\cdots-T_{i p}} \widehat{A}_{i_{p-1} 21} \xi_{1}\left(t^{j_{i_{p-1}}}\right), \\
\varphi_{2}(t)=\left(-\widehat{A}_{d i_{p-1} 22}\right)^{j_{i_{p-1}}-T_{i_{k}}-\cdots-T_{i p}} \widehat{A}_{d i_{p-1} 21} \xi_{1}\left(t^{j_{i_{p-1}}+1}\right)
\end{gathered}
$$

Therefore, from (3.15), (3.21), and (3.26), and noting $t^{T_{i_{k}}+\cdots+T_{i_{0}}} \in\left(-d_{1}-d_{2}, t_{0}\right]$, we obtain

$$
\left\|\xi_{2}(t)\right\| \leq \Delta_{1}+\Delta_{2}+\Delta_{3}+\Delta_{4}+\Delta_{5}
$$

where

$$
\begin{gathered}
\Delta_{1}=\left[\prod_{j=0}^{k}\left\|\left(\widehat{A}_{d i_{j} 22}\right)^{T_{i_{j}}}\right\|\right]\left\|x_{t_{0}}\right\|_{d_{1}+d_{2}{ }^{\prime}} \\
\Delta_{2}=\widehat{A}_{21} \sum_{j_{i_{k}}=0}^{T_{i_{k}}-1}\left\|\left(\widehat{A}_{d i_{k} 22}\right)^{j_{i_{k}}}\right\|\left\|\xi_{1}\left(t^{j_{i_{k}}}\right)\right\|, \\
\Delta_{3}=\widehat{A}_{d 21} \sum_{j_{i_{k}}=0}^{T_{i_{k}}-1}\left\|\left(\widehat{A}_{d i_{k} 22}\right)^{j_{i_{k}}}\right\|\left\|\xi_{1}\left(t^{j_{i_{k}}+1}\right)\right\|, \\
\Delta_{4}=\widehat{A}_{21} \sum_{p=1}^{k}\left\{\left[\prod_{q=p}^{k}\left\|\left(\widehat{A}_{d i_{q} 22}\right)^{T_{i_{q}}}\right\|\right]_{j_{i_{p-1}}=T_{i_{k}}+\cdots+T_{i_{p}}}^{T_{i_{k}}+\cdots+T_{i_{p-1}}-1}\left\|\varphi_{1}(t)\right\|\right\}, \\
\Delta_{5}=\widehat{A}_{d 21} \sum_{p=1}^{k}\left\{\left[\prod_{q=p}^{k}\left\|\left(\widehat{A}_{d i_{q} 22}\right)^{T_{i_{q}}}\right\|\right]_{j_{i_{p-1}}=T_{i_{k}+\cdots+T_{i_{p}}}^{T_{i_{k}}+\cdots+T_{i_{p-1}}-1}}^{\sum_{p}}\left\|\varphi_{2}(t)\right\|\right\} .
\end{gathered}
$$

Note

$$
t_{0} \geq t^{T_{i_{k}}+\cdots+T_{i_{0}}}=t-\sum_{j=0}^{T_{i_{k}}+\cdots+T_{i_{0}}-1} d\left(t^{j}\right) \geq t-\left(T_{i_{k}}+\cdots+T_{i_{0}}\right)\left(d_{1}+d_{2}\right)
$$


Using (3.19) and the relation $T_{a} \geq T_{a}^{*}=(\ln \beta) / \alpha$, the first term in (3.28) can be estimated as

$$
\begin{aligned}
\Delta_{1} & =\left[\prod_{j=0}^{k}\left\|\left(e^{(1 / 2) \alpha\left(d_{1}+d_{2}\right)} \widehat{A}_{d i_{j} 22}\right)^{T_{i_{j}}}\right\| e^{-(1 / 2) \alpha\left(T_{i_{k}}+\cdots+T_{i_{0}}\right)\left(d_{1}+d_{2}\right)}\right]\left\|x_{t_{0}}\right\|_{d_{1}+d_{2}} \\
& \leq\left[\prod_{j=0}^{k} \hbar_{i_{j}} e^{-\eta_{i_{j}} T_{i_{j}}}\right] e^{-(1 / 2) \alpha\left(t-t_{0}\right)}\left\|x_{t_{0}}\right\|_{d_{1}+d_{2}} \\
& \leq\left[\prod_{j=0}^{k} \hbar_{i_{j}} e^{-\eta_{i_{j}} T_{i_{j}}}\right] e^{-(1 / 2)\left(\alpha-(\ln \beta) / T_{a}\right)\left(t-t_{0}\right)}\left\|x_{t_{0}}\right\|_{d_{1}+d_{2}} \\
& :=\chi_{1} e^{-(1 / 2)\left(\alpha-(\ln \beta) / T_{a}\right)\left(t-t_{0}\right)}\left\|x_{t_{0}}\right\|_{d_{1}+d_{2}} .
\end{aligned}
$$

By (3.15), (3.12), (3.21), (3.20) and (3.19), we get

$$
\begin{aligned}
\left\|\left(\widehat{A}_{d i_{k} 22}\right)^{j_{i_{k}}}\right\|\left\|\xi_{1}\left(t^{j_{i_{k}}}\right)\right\| & \leq\left\|\left(\widehat{A}_{d i_{k} 22}\right)^{j_{i_{k}}}\right\| \sqrt{\frac{\lambda_{2}}{\lambda_{1}}} e^{-(1 / 2)\left(\alpha-(\ln \beta) / T_{a}\right)\left(t^{j_{k}}-t_{0}\right)}\left\|x_{t_{0}}\right\|_{d_{1}+d_{2}} \\
& \leq \sqrt{\frac{\lambda_{2}}{\lambda_{1}}}\left\|e^{(1 / 2) \alpha\left(d_{1}+d_{2}\right)}\left(\widehat{A}_{d i_{k} 22}\right)^{j_{i_{k}}}\right\| e^{-(1 / 2)\left(\alpha-(\ln \beta) / T_{a}\right)\left(t^{j_{i}-1}-t_{0}\right)}\left\|x_{t_{0}}\right\|_{d_{1}+d_{2}} \\
& \leq \cdots \leq \sqrt{\frac{\lambda_{2}}{\lambda_{1}}}\left\|\left(e^{(1 / 2) \alpha\left(d_{1}+d_{2}\right)} \widehat{A}_{d i_{k} 22}\right)^{j_{i_{k}}}\right\| e^{-(1 / 2)\left(\alpha-(\ln \beta) / T_{a}\right)\left(t^{0}-t_{0}\right)}\left\|x_{t_{0}}\right\|_{d_{1}+d_{2}} \\
& \leq \sqrt{\frac{\lambda_{2}}{\lambda_{1}}}\left(\hbar_{i_{k}} e^{-\eta_{i_{k}} j_{i_{k}}}\right) e^{-(1 / 2)\left(\alpha-(\ln \beta) / T_{a}\right)\left(t-t_{0}\right)}\left\|x_{t_{0}}\right\|_{d_{1}+d_{2}} .
\end{aligned}
$$

Then, the second term in (3.28) can be estimated as

$$
\begin{aligned}
\Delta_{2} & \leq \widehat{A}_{21} \sqrt{\frac{\lambda_{2}}{\lambda_{1}}}\left[\sum_{j_{i_{k}}=0}^{T_{i_{k}}-1} \hbar_{i_{k}} e^{-\eta_{i_{k}} j_{i_{k}}}\right] e^{-(1 / 2)\left(\alpha-(\ln \beta) / T_{a}\right)\left(t-t_{0}\right)}\left\|x_{t_{0}}\right\|_{d_{1}+d_{2}} \\
& \leq \hbar_{i_{k}} \widehat{A}_{21} \sqrt{\frac{\lambda_{2}}{\lambda_{1}}} \frac{e^{\eta_{i_{k}}}}{e^{\eta_{i_{k}}-1}} e^{-(1 / 2)\left(\alpha-(\ln \beta) / T_{a}\right)\left(t-t_{0}\right)}\left\|x_{t_{0}}\right\|_{d_{1}+d_{2}} \\
& :=\chi_{2} e^{-(1 / 2)\left(\alpha-(\ln \beta) / T_{a}\right)\left(t-t_{0}\right)}\left\|x_{t_{0}}\right\|_{d_{1}+d_{2}} .
\end{aligned}
$$

Similarly, the third term in (3.28) can be bounded by

$$
\begin{aligned}
\Delta_{3} & \leq \hbar_{i_{k}} e^{(1 / 2) \alpha\left(d_{1}+d_{2}\right)} \widehat{A}_{d 21} \sqrt{\frac{\lambda_{2}}{\lambda_{1}}} \frac{e^{\eta_{i_{k}}}}{e^{\eta_{i_{k}}-1}} e^{-(1 / 2)\left(\alpha-(\ln \beta) / T_{a}\right)\left(t-t_{0}\right)}\left\|x_{t_{0}}\right\|_{d_{1}+d_{2}} \\
& :=\chi_{3} e^{-(1 / 2)\left(\alpha-(\ln \beta) / T_{a}\right)\left(t-t_{0}\right)}\left\|x_{t_{0}}\right\|_{d_{1}+d_{2}} .
\end{aligned}
$$


In addition, following a similar deduction as that in (3.32), we obtain

$$
\left\|\varphi_{1}(t)\right\| \leq \sqrt{\frac{\lambda_{2}}{\lambda_{1}}}\left(e^{(1 / 2) \alpha\left(d_{1}+d_{2}\right)}\right)^{T_{i_{k}}+\cdots+T_{i p}}\left(\hbar_{i_{p-1}} e^{-\eta_{i_{p-1}}\left(j_{i_{p-1}}-T_{i_{k}}-\cdots-T_{i_{p}}\right)}\right) e^{-(1 / 2)\left(\alpha-(\ln \beta) / T_{a}\right)\left(t-t_{0}\right)}\left\|x_{t_{0}}\right\|_{d_{1}+d_{2}} .
$$

Then, considering this and (3.19), the fourth term in (3.28) can be estimated as

$$
\begin{aligned}
\Delta_{4} \leq & \widehat{A}_{21} \sqrt{\frac{\lambda_{2}}{\lambda_{1}}} \sum_{p=1}^{k}\left\{\left[\prod_{q=p}^{k}\left\|\left(e^{(1 / 2) \alpha\left(d_{1}+d_{2}\right)} \widehat{A}_{d i_{q} 22}\right)^{T_{i_{q}}}\right\|\right]_{j_{i_{p-1}}=T_{i_{k}}+\cdots+T_{i p}}^{T_{i_{k}}+\cdots+T_{i_{p-1}}-1} \hbar_{i_{p-1}} e^{-\eta_{i_{p-1}}\left(j_{i_{p-1}}-T_{i_{k}}-\cdots-T_{i_{p}}\right)}\right\} \\
& \times e^{-(1 / 2)\left(\alpha-(\ln \beta) / T_{a}\right)\left(t-t_{0}\right)}\left\|x_{t_{0}}\right\|_{d_{1}+d_{2}} \\
\leq & \widehat{A}_{21} \sqrt{\frac{\lambda_{2}}{\lambda_{1}}} \sum_{p=1}^{k}\left\{\hbar_{i_{p-1}}\left[\prod_{q=p}^{k} \hbar_{i_{q}} e^{-\eta_{i q} T_{i_{q}}}\right] \frac{e^{\eta_{i_{p-1}}}}{\left.e^{\eta_{i_{p-1}}-1}\right\} e^{-(1 / 2)\left(\alpha-(\ln \beta) / T_{a}\right)\left(t-t_{0}\right)}\left\|x_{t_{0}}\right\|_{d_{1}+d_{2}}}\right. \\
:= & X_{4} e^{-(1 / 2)\left(\alpha-(\ln \beta) / T_{a}\right)\left(t-t_{0}\right)}\left\|x_{t_{0}}\right\|_{d_{1}+d_{2}} .
\end{aligned}
$$

Similarly, the fifth term in (3.28) can be bounded by

$$
\begin{aligned}
\Delta_{5} & \leq e^{(1 / 2) \alpha\left(d_{1}+d_{2}\right)} \widehat{A}_{d 21} \sqrt{\frac{\lambda_{2}}{\lambda_{1}}} \sum_{p=1}^{k}\left\{\hbar_{i_{p-1}}\left[\prod_{q=p}^{k} \hbar_{i_{q}} e^{-\eta_{i_{q}} T_{i_{q}}}\right] \frac{e^{\eta_{i_{p-1}}}}{e^{\eta_{p_{p-1}}}-1}\right\} e^{-(1 / 2)\left(\alpha-(\ln \beta) / T_{a}\right)\left(t-t_{0}\right)}\left\|x_{t_{0}}\right\|_{d_{1}+d_{2}} \\
& :=X_{5} e^{-(1 / 2)\left(\alpha-(\ln \beta) / T_{a}\right)\left(t-t_{0}\right)}\left\|x_{t_{0}}\right\|_{d_{1}+d_{2}} .
\end{aligned}
$$

Therefore, using (3.31) and (3.33)-(3.37), $\left\|\xi_{2}(t)\right\|$ can be estimated as

$$
\left\|\xi_{2}(t)\right\| \leq\left(\chi_{1}+\chi_{2}+\chi_{3}+\chi_{4}+\chi_{5}\right) e^{-(1 / 2)\left(\alpha-(\ln \beta) / T_{a}\right)\left(t-t_{0}\right)}\left\|x_{t_{0}}\right\|_{d_{1}+d_{2}} .
$$

Combining (3.15), (3.12) and (3.38) yields that system (2.4) is exponentially stable for any switching sequence $\mathcal{S}$ with average dwell time $T_{a} \geq T_{a}^{*}=(\ln \beta) / \alpha$. This completes the proof.

Remark 3.2. In terms of LMIs, Theorem 3.1 presents a delay-range-dependent exponential admissibility condition for the switched singular systems with interval time-varying delay. It is noted that this condition is obtained by using the integral inequality (Lemma 2.4); no additional free-weighting matrices are introduced to deal with the cross-term. Therefore, the condition proposed here involves much less decision variables than those obtained by using the free-weighting matrices method $[16,19,21,22]$ if the same Lyapunov function is chosen.

Remark 3.3. Equation (3.26) plays an important role in analyzing the exponential stability of the algebraic subsystem, which can be seen as a generalization of the iterative equation in [37] for nonswitched singular time-delay system to switched case. 
Remark 3.4. If $\beta=1$ in $T_{a} \geq T_{a}^{*}=(\ln \beta) / \alpha$, which leads to $P_{i 11} \equiv P_{j 11}, Q_{i l} \equiv Q_{j l}, Z_{i l} \equiv Z_{j l}$, $l=1,2$, for all $i, j \in O$, and $T_{a}^{*}=0$, then system (2.4) possesses a common Lyapunov function and the switching signals can be arbitrary.

In the following, we are to deal with the design problem of DOF controller for the SSTD system (2.1). Applying the DOF controller (2.5) to system (2.1) gives the following closed-loop system

$$
\bar{E} \dot{\eta}(t)=\bar{A}_{\sigma(t)} \eta(t)+\bar{A}_{d \sigma(t)} \eta(t-d(t))
$$

where $\eta(t)=\left[x^{T}(t) x_{c}^{T}(t)\right]^{T}$, and

$$
\begin{gathered}
\bar{E}=\left[\begin{array}{cc}
E & 0 \\
0 & E_{c}
\end{array}\right], \quad \bar{A}_{\sigma(t)}=\left[\begin{array}{cc}
A_{\sigma(t)}+B_{\sigma(t)} D_{c \sigma(t)} C_{\sigma(t)} & B_{\sigma(t)} C_{c \sigma(t)} \\
B_{c \sigma(t)} C_{\sigma(t)} & A_{c \sigma(t)}
\end{array}\right] \\
\bar{A}_{d \sigma(t)}=\left[\begin{array}{cc}
A_{d \sigma(t)}+B_{\sigma(t)} D_{c \sigma(t)} C_{d \sigma(t)} & 0 \\
B_{c \sigma(t)} C_{d \sigma(t)} & 0
\end{array}\right] .
\end{gathered}
$$

The following Theorem presents a sufficient condition for solvability of the DOF controller design problem for system (2.1).

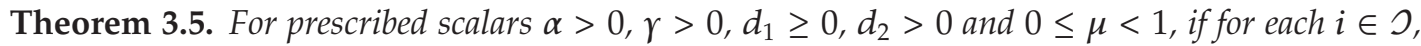
and given scalars $\xi_{i 1}$ and $\xi_{i 2}$, there exist matrices $Y_{i}, \widehat{A}_{c i}, \widehat{B}_{c i}, \widehat{C}_{c i}, \widehat{D}_{c i}, Q_{i l 11}, Q_{i l 12}, Q_{i l 22}, Z_{i l 11}, Z_{i l 12}$, $Z_{i l 22}, l=1,2$, and $R_{i}$ and $U_{i}$ of the following form

$$
R_{i}=\left[\begin{array}{cc}
R_{i 11} & 0 \\
R_{i 21} & R_{i 22}
\end{array}\right], \quad U_{i}=\left[\begin{array}{cc}
U_{i 11} & 0 \\
U_{i 21} & U_{i 22}
\end{array}\right]
$$

with $R_{i 11} \in \mathbf{R}^{r \times r}, U_{i 11} \in \mathbf{R}^{r \times r}, R_{i 11}>0, U_{i 11}>0, R_{i 22} \in \mathbf{R}^{(n-r) \times(n-r)}, U_{i 22} \in \mathbf{R}^{(n-r) \times(n-r)}$, and $R_{i 22}$ and $U_{i 22}$ being invertible, such that

$$
\begin{aligned}
& {\left[\begin{array}{cccccccc}
\Upsilon_{i 11} & \Upsilon_{i 12} & \Upsilon_{i 13} & \Upsilon_{i 14} & \Upsilon_{i 15} & 0 & c_{1} E^{T} Z_{i 111} E & c_{1} E^{T} Z_{i 112} E \\
* & \Upsilon_{i 22} & \Upsilon_{i 23} & \Upsilon_{i 24} & \Upsilon_{i 25} & 0 & c_{1} E^{T} Z_{i 112}^{T} E & c_{1} E^{T} Z_{i 122} E \\
* & * & \Upsilon_{i 33} & \Upsilon_{i 34} & \Upsilon_{i 35} & 0 & 0 & 0 \\
* & * & * & \Upsilon_{i 44} & \Upsilon_{i 45} & 0 & 0 & 0 \\
* & * & * & * & \Upsilon_{i 55} & \Upsilon_{i 56} & c_{2} E^{T} Z_{i 211} E & c_{2} E^{T} Z_{i 212} E \\
* & * & * & * & * & \Upsilon_{i 66} & c_{2} E^{T} Z_{i 212}^{T} E & c_{2} E^{T} Z_{i 222} E \\
* & * & * & * & * & * & \Upsilon_{i 77} & \Upsilon_{i 78} \\
* & * & * & * & * & * & * & \Upsilon_{i 88}
\end{array}\right]<0,} \\
& \bar{Q}_{i l}=\left[\begin{array}{cc}
Q_{i l 11} & Q_{i l 12} \\
* & Q_{i l 22}
\end{array}\right]>0, \quad \bar{Z}_{i l}=\left[\begin{array}{cc}
Z_{i l 11} & Z_{i l 12} \\
* & Z_{i l 22}
\end{array}\right]>0, \quad l=1,2,
\end{aligned}
$$


where $\Upsilon_{i 11}=\operatorname{Sym}\left\{\xi_{i 1} A_{i}+\xi_{i 1} B_{i} \widehat{D}_{c i} C_{i}+\widehat{B}_{c i} C_{i}\right\}+\sum_{l=1}^{2} Q_{i l 11}+\alpha E^{T} R_{i}-c_{1} E^{T} Z_{i 111} E, \Upsilon_{i 12}=\widehat{A}_{c i}+\xi_{i 1} B_{i} \widehat{C}_{c i}+$ $\xi_{i 2} A_{i}^{T}+\xi_{i 2} C_{i}^{T} \widehat{D}_{c i}^{T} B_{i}^{T}+C_{i}^{T} \widehat{B}_{c i}^{T}+\sum_{l=1}^{2} Q_{i l 12}-c_{1} E^{T} Z_{i 112} E, \Upsilon_{i 13}=-\xi_{i 1} I+R_{i}^{T}+\xi_{i 1} A_{i}^{T}+\xi_{i 1} C_{i}^{T} \widehat{D}_{c i}^{T} B_{i}^{T}+$ $C_{i}^{T} \widehat{B}_{c i}^{T} \Upsilon_{i 14}=-\Upsilon_{i}+\xi_{i 2} A_{i}^{T}+\xi_{i 2} C_{i}^{T} \widehat{D}_{c i}^{T} B_{i}^{T}+C_{i}^{T} \widehat{B}_{c i}^{T}, \Upsilon_{i 15}=\Upsilon_{i 35}=\xi_{i 1} A_{d i}+\xi_{i 1} B_{i} \widehat{D}_{c i} C_{d i}+\widehat{B}_{c i} C_{d i}$, $\Upsilon_{i 22}=\operatorname{Sym}\left\{\widehat{A}_{c i}+\xi_{i 2} B_{i} \widehat{C}_{c i}\right\}+\sum_{l=1}^{2} Q_{i l 22}+\alpha E^{T} U_{i}-c_{1} E^{T} Z_{i 122} E, \Upsilon_{i 23}=-\xi_{i 2} I+\widehat{A}_{c i}^{T}+\xi_{i 1} \widehat{C}_{c i}^{T} B_{i}^{T}$, $\Upsilon_{i 24}=-\Upsilon_{i}+\widehat{A}_{c i}^{T}+\xi_{i 2} \widehat{C}_{c i}^{T} B_{i}^{T}+U_{i}^{T}, \Upsilon_{i 25}=\Upsilon_{i 45}=\xi_{i 2} A_{d i}+\xi_{i 2} B_{i} \widehat{D}_{c i} C_{d i}+\widehat{B}_{c i} C_{d i}, \Upsilon_{i 33}=-2 \xi_{i 1} I+d_{1}^{2} Z_{i 111}+$ $d_{2}^{2} e^{\alpha d_{1}} Z_{i 211}, \Upsilon_{i 34}=-\xi_{i 2} I-Y_{i}+d_{1}^{2} Z_{i 112}+d_{2}^{2} e^{\alpha d_{1}} Z_{i 212}, \Upsilon_{i 44}=-\operatorname{Sym}\left\{Y_{i}\right\}+d_{1}^{2} Z_{i 122}+d_{2}^{2} e^{\alpha d_{1}} Z_{i 222}$, $\Upsilon_{i 55}=-(1-\mu) e^{-\alpha\left(d_{1}+d_{2}\right)} Q_{i 211}-c_{2} E^{T} Z_{i 211} E, \Upsilon_{i 56}=-(1-\mu) e^{-\alpha\left(d_{1}+d_{2}\right)} Q_{i 212}-c_{2} E^{T} Z_{i 212} E, \Upsilon_{i 66}=$ $-(1-\mu) e^{-\alpha\left(d_{1}+d_{2}\right)} Q_{i 222}-c_{2} E^{T} Z_{i 222} E, \Upsilon_{i 77}=-(1-\mu) e^{-\alpha d_{1}} Q_{i 111}-c_{1} E^{T} Z_{i 111} E-c_{2} E^{T} Z_{i 211} E, \Upsilon_{i 78}=$ $-(1-\mu) e^{-\alpha d_{1}} Q_{i 112}-c_{1} E^{T} Z_{i 112} E-c_{2} E^{T} Z_{i 212} E, \Upsilon_{i 88}=-(1-\mu) e^{-\alpha d_{1}} Q_{i 122}-c_{1} E^{T} Z_{i 122} E-c_{2} E^{T} Z_{i 222} E$. Then, there exists a DOF controller in the form of (2.5), such that system (3.39) is exponentially admissible for any switching sequence $\mathcal{S}$ with average dwell time $T_{a} \geq T_{a}^{*}=\ln \beta / \alpha$, where $\beta \geq 1$ satisfies

$$
R_{i 11} \leq \beta R_{j 11}, \quad U_{i 11} \leq \beta U_{j 11}, \quad \bar{Q}_{i l} \leq \beta \bar{Q}_{j l}, \quad \bar{Z}_{i l} \leq \beta \bar{Z}_{j l}, \quad l=1,2, \quad \forall i, j \in \mathcal{O} .
$$

Moreover, a desired DOF controller realisation is given by

$$
A_{c i}=Y_{i}^{-1} \widehat{A}_{c i}, \quad B_{c i}=Y_{i}^{-1} \widehat{B}_{c i}, \quad C_{c i}=\widehat{C}_{c i}, \quad D_{c i}=\widehat{D}_{c i}, \quad \forall i \in \supset
$$

Proof. From Theorem 3.1, we known that system (3.39) is exponentially admissible for any switching sequence $\mathcal{S}$ with average dwell time $T_{a} \geq T_{a}^{*}=(\ln \beta) / \alpha$, where $\beta \geq 1$ satisfying (3.3), if for each $i \in \mathcal{O}$, there exist matrices $\bar{Q}_{i l}>0, \bar{Z}_{i l}>0, l=1,2$, and $\bar{P}_{i}$ with the form of (3.1) such that the inequality (3.2) with $E, A_{i}$ and $A_{d i}$ instead of $\bar{E}, \bar{A}_{i}$ and $\bar{C}_{i}$, respectively, holds. By decomposing $\Phi_{i}$ in (3.2), we obtain that for each $i \in \mathcal{J}$

$$
\Phi_{i}=\Pi_{i} \Lambda_{i} \Pi_{i}^{T}<0,
$$

where $\bar{J}_{i}$ is any invertible matrix with compatible dimension, and

$$
\Pi_{i}=\left[\begin{array}{ccc}
I & 0 & 0 \\
\bar{A}_{i} & \bar{A}_{d i} & 0 \\
0 & I & 0 \\
0 & 0 & I
\end{array}\right]^{T}, \quad \Lambda_{i}=\left[\begin{array}{cccc}
\Lambda_{i 11} & \bar{P}_{i}^{T}-\bar{J}_{i}+\bar{A}_{i}^{T} \bar{J}_{i}^{T} & \bar{J}_{i} \bar{A}_{d i} & c_{1} \bar{E}^{T} \bar{Z}_{i 1} \bar{E} \\
* & -\bar{J}_{i}-\bar{J}_{i}^{T}+\bar{U}_{i} & \bar{J}_{i} \bar{A}_{d i} & 0 \\
* & * & \Lambda_{i 33} & c_{2} \bar{E}^{T} \bar{Z}_{i 2} \bar{E} \\
* & * & * & \Lambda_{i 44}
\end{array}\right]
$$

with $\Lambda_{i 11}=\operatorname{Sym}\left\{\bar{J}_{i} \bar{A}_{i}\right\}+\sum_{l=1}^{2} \bar{Q}_{i l}+\alpha \bar{E}_{i}^{T} \bar{P}_{i}-c_{1} \bar{E}^{T} \bar{Z}_{i 1} \bar{E}, \Lambda_{i 33}=-(1-\mu) e^{-\alpha\left(d_{1}+d_{2}\right)} \bar{Q}_{i 2}-c_{2} \bar{E}^{T} \bar{Z}_{i 2} \bar{E}$, and $\Lambda_{i 44}=-e^{-\alpha d_{1}} \bar{Q}_{i 1}-c_{1} \bar{E}^{T} \bar{Z}_{i 1} \bar{E}-c_{2} \bar{E}^{T} \bar{Z}_{i 2} \bar{E}$. Hence, $\Phi_{i}<0$ holds if

$$
\Lambda_{i}<0
$$

Let $\bar{E}=\operatorname{diag}\{E, E\}$. For each $i \in \mathcal{O}$, define

$$
\bar{P}_{i}=\operatorname{diag}\left\{R_{i}, U_{i}\right\}
$$


By (2.3) and (3.41), we have

$$
E^{T} R_{i}=R_{i}^{T} E \geq 0, \quad E^{T} U_{i}=U_{i}^{T} E \geq 0,
$$

which combining (3.49) yields

$$
\bar{E}^{T} \bar{P}_{i}=\bar{P}_{i}^{T} \bar{E} \geq 0
$$

Then, from (3.41), (3.44) and (3.51), it can be deduced that

$$
\bar{E}^{T} \bar{P}_{i} \leq \beta \bar{E}^{T} \bar{P}_{j}, \quad \forall i, j \in \supset .
$$

Denote

$$
\bar{J}_{i}=\left[\begin{array}{ll}
\xi_{i 1} I & Y_{i} \\
\xi_{i 2} I & Y_{i}
\end{array}\right] .
$$

Substituting (3.43), (3.49) and (3.53) into (3.48), and defining

$$
\widehat{A}_{c i}=Y_{i} A_{c i}, \quad \widehat{B}_{c i}=Y_{i} B_{c i}, \quad \widehat{C}_{c i}=C_{c i}, \quad \widehat{D}_{c i}=D_{c i}, \quad \forall i \in \mathcal{O}
$$

we can easily obtain (3.42). This completes the proof.

Remark 3.6. Note that condition $\Phi_{i}$ of Theorem 3.1 involves some product terms between the Lyapunov matrices and the system matrices, which complicates the DOF control synthesis problem. To solve this problem, in the proof of Theorem 3.5, we have made a decoupling between the Lyapunov matrices and the system matrices by introducing a slack matrix $\bar{J}_{i}$ in condition $\Lambda_{i}$. Compared with the variable change method used in $[39,40]$, the decoupling technique proposed here simplifies the DOF controller design problem greatly, which decreases the conservatism in some sense.

Remark 3.7. Scalars $\xi_{i 1}$ and $\xi_{i 2}, i \in \mathcal{O}$, in Theorem 3.5 are tuning parameters which need to be specified first. The optimal values of these parameters can be found by applying some optimization algorithms such as the program fminsearch in the optimization toolbox of MATLAB, the branch-and-band algorithm [41].

Remark 3.8. It is noted that in this paper, the derivative matrix $E$ is assumed to be switchmode-independent. If $E$ is also switch-mode-dependent, then $E$ is changed to $E_{i}, i \in \mathcal{O}$. In this case, the transformation matrices $P$ and $Q$ should become $P_{i}$ and $Q_{i}$ so that $P_{i} E_{i} Q_{i}=$ $\operatorname{diag}\left\{I_{r_{i}}, 0\right\}$, and the state of the transformed system becomes $\tilde{x}(t)=Q_{i}^{-1}(t)=\left[\begin{array}{ll}\tilde{x}_{i 1}^{T}(t) & \tilde{x}_{i 2}^{T}(t)\end{array}\right]^{T}$ with $\tilde{x}_{i 1}^{T}(t) \in \mathbf{R}^{r_{i}}$ and $\tilde{x}_{i 1}^{T}(t) \in \mathbf{R}^{n-r_{i}}$, which implies that there does not exist one common state space coordinate basis for all subsystems. Then, some assumptions for $E_{i}$ (e.g., $E_{i}, i \in \mathcal{O}$, have the same right zero subspace [27]) should be made so that $Q_{i}$ remains the same; in this case, the method presented in this paper is also valid. How to investigate the general SSTD system with $E$ being switch-mode-dependent is an interesting problem for future work via other approaches. 
Table 1: Comparison of allowable upper bound $\tilde{d}_{2}$ for different $d_{1}$ in Example 4.1.

\begin{tabular}{|c|c|c|c|c|}
\hline Methods & & $\widetilde{d}_{2}$ & & Number of variables \\
\hline Lemma 1 [21] & $1.130\left(d_{1}=0.1\right)$ & $1.099\left(d_{1}=0.3\right)$ & $1.084\left(d_{1}=0.7\right)$ & 84 \\
\hline Theorem 1 [22] & $1.130\left(d_{1}=0.1\right)$ & $1.099\left(d_{1}=0.3\right)$ & $1.084\left(d_{1}=0.7\right)$ & 84 \\
\hline Theorem 3.1 & $1.134\left(d_{1}=0.1\right)$ & $1.133\left(d_{1}=0.3\right)$ & $1.133\left(d_{1}=0.7\right)$ & 30 \\
\hline
\end{tabular}

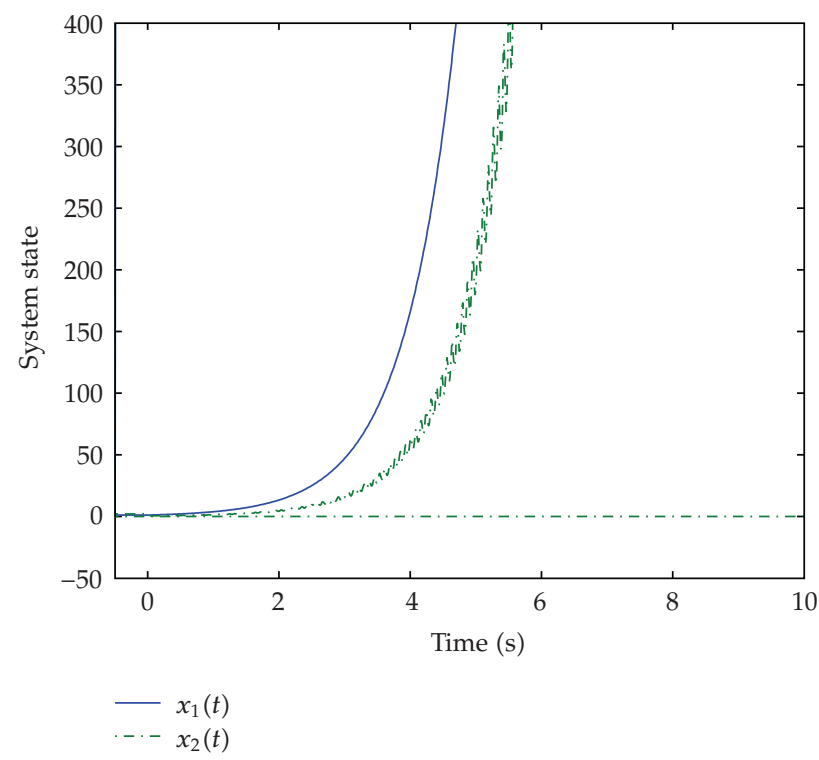

Figure 1: State trajectories of the open-loop subsystem 1.

\section{Numerical Examples}

In this section, some numerical examples are presented to demonstrate the effectiveness of the proposed methods.

Example 4.1. Consider the switched system (2.4) with $E=I, N=2$ (e.g., there are two subsystems) and the following parameters, which are borrowed from [21]:

$$
A_{1}=\left[\begin{array}{cc}
-2 & 0 \\
0 & -0.9
\end{array}\right], \quad A_{d 1}=\left[\begin{array}{cc}
-1 & 0 \\
0 & -1
\end{array}\right], \quad A_{2}=\left[\begin{array}{cc}
-2 & 0 \\
0 & -0.7
\end{array}\right], \quad A_{d 2}=\left[\begin{array}{cc}
-1 & 0 \\
-1 & -1
\end{array}\right] .
$$

For $\mu=0.4, \alpha=0.5$ and $\beta=1.1$, employing the LMIs in [21, 22] and those in Theorem 3.1 yields an allowable upper bound $\tilde{d}_{2}$ (in this paper $\tilde{d}_{2}=d_{1}+d_{2}$ ) of the delay $d(t)$ that guarantees the stability of system (2.4). Table 1 shows the values of the upper bound for various $d_{1}$ and the number of involved variables by using different methods. It is easily seen from Table 1 that Theorem 3.1 of this paper not only provides better results than those criteria in $[21,22]$ but also reduces the computational overhead to some extent. 


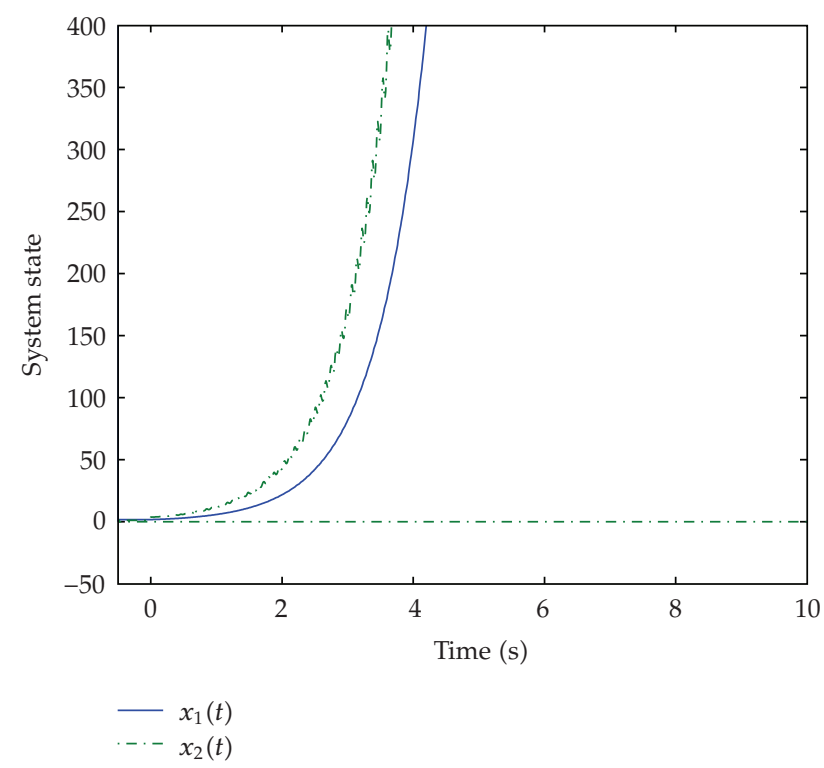

Figure 2: State trajectories of the open-loop subsystem 2.

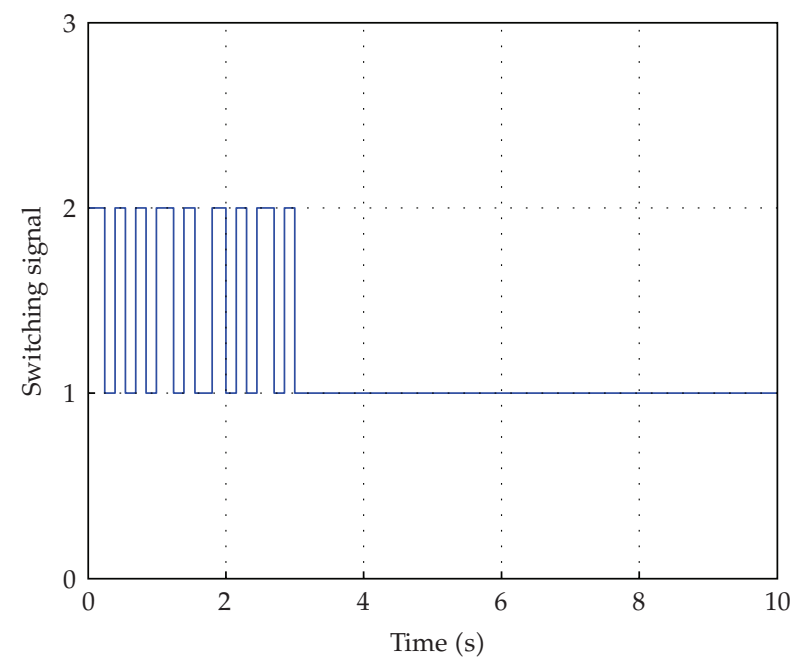

Figure 3: Switching signal with the average dwell time $T_{a}>0.13$.

Example 4.2. Consider the switched system (2.4) with $N=2$ and the related parameters are given as follows:

$$
\begin{gathered}
E=\left[\begin{array}{ll}
1 & 0 \\
0 & 0
\end{array}\right], \quad A_{1}=\left[\begin{array}{cc}
0.73 & 0 \\
0 & -1
\end{array}\right], \quad A_{d 1}=\left[\begin{array}{cc}
-1.1 & 1 \\
0 & 0.5
\end{array}\right], \\
A_{2}=\left[\begin{array}{cc}
0.4 & 0 \\
-0.1 & -1
\end{array}\right], \quad A_{d 2}=\left[\begin{array}{cc}
-1 & 0.1 \\
0 & 0.1
\end{array}\right]
\end{gathered}
$$




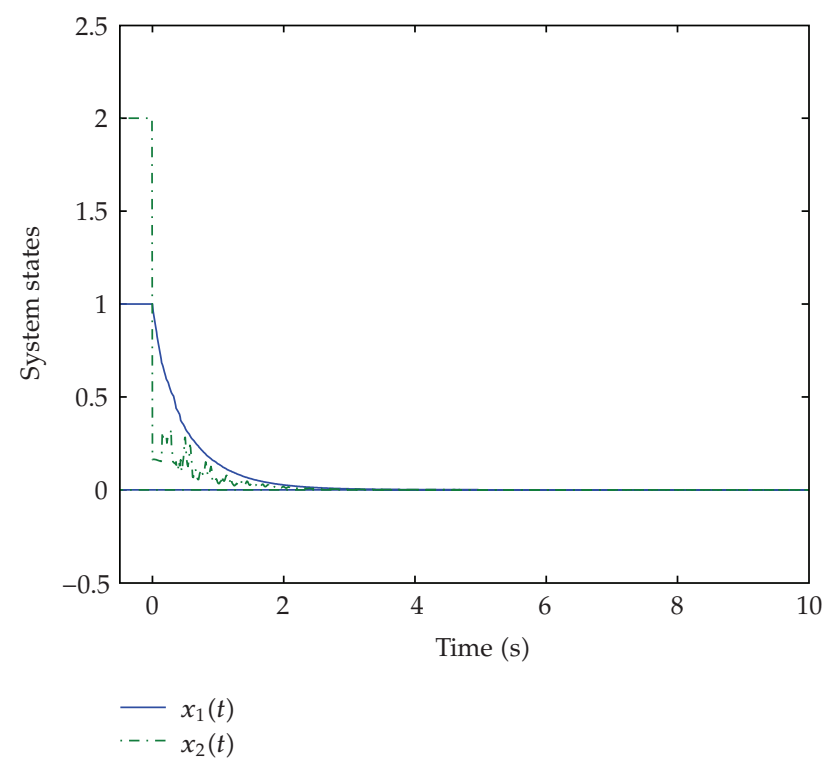

Figure 4: State trajectories of the closed-loop system under DOF control.

and $d_{1}=0.1, d_{2}=0.2, \mu=0.4$ and $\alpha=0.5$. It can be verified that both of the above two subsystems are stable. Let $\beta=1$; it can be found that there is no feasible solution to this case, which implies that there is no common Lyapunov function for the above two subsystems (see Remark 3.4). Now, we consider the average dwell time scheme, and set $\beta=1.2$. Solving the LMIs (3.2) gives the following solutions:

$$
\begin{aligned}
& P_{1}=\left[\begin{array}{cc}
19.7719 & 0 \\
17.9611 & 819.3011
\end{array}\right], \quad Q_{11}=\left[\begin{array}{cc}
0.7438 & -1.2478 \\
-1.2478 & 68.3107
\end{array}\right], \quad Q_{12}=10^{3} \times\left[\begin{array}{cc}
0.0002 & 0.0102 \\
0.0102 & 1.0735
\end{array}\right], \\
& Z_{11}=\left[\begin{array}{cc}
624.4425 & 0.8810 \\
0.8810 & 379.5848
\end{array}\right], \quad Z_{12}=\left[\begin{array}{cc}
332.4246 & -0.4902 \\
-0.4902 & 373.4311
\end{array}\right], \\
& P_{2}=\left[\begin{array}{cc}
23.3711 & 0 \\
-26.9375 & 642.4532
\end{array}\right], \quad Q_{21}=\left[\begin{array}{cc}
0.7501 & -0.8269 \\
-0.8269 & 66.5062
\end{array}\right], \quad Q_{22}=\left[\begin{array}{cc}
0.2201 & 8.9316 \\
8.9316 & 917.3497
\end{array}\right], \\
& Z_{21}=\left[\begin{array}{cc}
560.8384 & 0.7643 \\
0.7643 & 379.5975
\end{array}\right], \quad Z_{22}=\left[\begin{array}{cc}
294.2097 & -0.4003 \\
-0.4003 & 373.4858
\end{array}\right]
\end{aligned}
$$

which means that the above switched system is exponentially admissible. Moreover, by further analysis, it can be found that the allowable minimum of $\beta$ is $\beta_{\min }=1.046$ when $\alpha=0.5$; in this case $T_{a}^{*}=\left(\ln \beta_{\min }\right) / \alpha=0.0899$. 
Example 4.3. Consider the switched system (2.1) with $N=2$ and

$$
\begin{gathered}
E=\left[\begin{array}{ll}
1 & 0 \\
0 & 0
\end{array}\right], \\
A_{1}=\left[\begin{array}{cc}
0.9 & 0 \\
1 & -5
\end{array}\right], \quad A_{d 1}=\left[\begin{array}{cc}
0.5 & 0.1 \\
1 & 0.1
\end{array}\right], \\
B_{1}=\left[\begin{array}{l}
0.3 \\
0.1
\end{array}\right], \quad C_{1}=\left[\begin{array}{ll}
0.3 & 0.1
\end{array}\right], \quad C_{d 1}=\left[\begin{array}{ll}
0.1 & 0.1
\end{array}\right], \\
A_{2}=\left[\begin{array}{cc}
0.5 & 0.1 \\
2 & -5
\end{array}\right], \quad A_{d 2}=\left[\begin{array}{ll}
0.2 & 0.5 \\
1.5 & 0.1
\end{array}\right], \quad B_{2}=\left[\begin{array}{l}
0.4 \\
0.1
\end{array}\right], \\
C_{2}=\left[\begin{array}{ll}
0.1 & 0.3
\end{array}\right], \quad C_{d 2}=\left[\begin{array}{ll}
0.1 & 0.1
\end{array}\right],
\end{gathered}
$$

and $d(t)=0.3+0.2 \sin (1.5 t)$. A simple calculation yields $d_{1}=0.1, d_{2}=0.4$ and $\mu=0.3$. By simulation, it can be checked that both of the above two subsystems with $u(t)=0$ are unstable, and the state responses of the corresponding open-loop systems are shown in Figures 1 and 2, respectively, with the initial condition given by $\phi(t)=\left[\begin{array}{ll}1 & 2\end{array}\right]^{T}, t \in[-0.5,0]$. In view of this, our goal is to design a DOF control $u(t)$ in the form of (2.5) such that the closed-loop system is exponentially admissible.

Set $\alpha=0.5, \beta=1.05$ (thus $T_{a} \geq T_{a}^{*}=(\ln \beta) / \alpha=0.0976$ ), and choose $\xi_{11}=0.9255$, $\xi_{12}=0.0067, \xi_{13}=0.9811, \xi_{14}=0.0016$. Solving the LMIs (3.41)-(3.44), the corresponding gain matrices of the DOF controller are computed as

$$
\begin{gathered}
A_{c 1}=\left[\begin{array}{cc}
-26.3829 & -0.4700 \\
-0.8920 & -0.8529
\end{array}\right], \quad A_{c 2}=\left[\begin{array}{cc}
-67.7480 & 0.6423 \\
9.5961 & -0.8803
\end{array}\right], \quad B_{c 1}=\left[\begin{array}{c}
0.6811 \\
-0.2272
\end{array}\right], \\
B_{c 2}=\left[\begin{array}{c}
4.0654 \\
-1.1668
\end{array}\right], \quad C_{c 1}=\left[\begin{array}{lll}
33.6391 & 0.1324
\end{array}\right], \quad C_{c 2}=[23.4740-0.5180] \\
D_{c 1}=-22.4825, \quad D_{c 2}=-17.9093
\end{gathered}
$$

To show the effectiveness of the obtained DOF controller, giving a random switching signal with the average dwell time $T_{a} \geq 0.13$ as shown in Figure 3, we get the state trajectories of the closed-loop system as shown in Figure 4, for the given initial condition $\phi(t)=\left[\begin{array}{ll}1 & 2\end{array}\right]^{T}$, $t \in[-0.5,0]$. It is clear that the designed controller is feasible and ensures the stability of the closed-loop system despite the switching and the time-varying delay.

\section{Conclusions}

In this paper, the problems of exponential admissibility and DOF control for a class of continuous-time switched singular systems with interval time-varying delay have been 
investigated. A class of switching signals has been identified for the switched singular timedelay systems to be exponentially admissible under the average dwell time scheme. The DOF controller has been designed, and the corresponding solvability condition has been established by using the LMI technique. Numerical examples have been provided to illustrate the effectiveness of the proposed methods.

\section{Acknowledgments}

This paper was supported by the National Natural Science Foundation of China (60904020, 61004032), the Research Fund for the Doctoral Program of Higher Education of China (20090092120027), and the Natural Science Foundation of Jiangsu Province (BK2010200).

\section{References}

[1] D. Liberzon and A. S. Morse, "Basic problems in stability and design of switched systems," IEEE Control Systems Magazine, vol. 19, no. 5, pp. 59-70, 1999.

[2] R. A. Decarlo, M. S. Branicky, S. Pettersson, and B. Lennartson, "Perspectives and results on the stability and stabilizability of hybrid systems," Proceedings of the IEEE, vol. 88, no. 7, pp. 1069-1082, 2000.

[3] Z. Sun and S. S. Ge, "Analysis and synthesis of switched linear control systems," Automatica, vol. 41, no. 2, pp. 181-195, 2005.

[4] H. Lin and P. J. Antsaklis, "Stability and stabilizability of switched linear systems: a survey of recent results," IEEE Transactions on Automatic Control, vol. 54, no. 2, pp. 308-322, 2009.

[5] M. S. Branicky, "Multiple Lyapunov functions and other analysis tools for switched and hybrid systems," IEEE Transactions on Automatic Control, vol. 43, no. 4, pp. 475-482, 1998.

[6] M. A. Wicks, P. Peleties, and R. A. DeCarlo, "Construction of piecewise Lyapunov functions for stabilizing switched systems," in Proceedings of the 33rd IEEE Conference on Decision and Control (CDC '94), pp. 3492-3497, December 1994.

[7] J. Daafouz, P. Riedinger, and C. Iung, "Stability analysis and control synthesis for switched systems: a switched Lyapunov function approach," IEEE Transactions on Automatic Control, vol. 47, no. 11, pp. 1883-1887, 2002.

[8] M. Wicks, P. Peleties, and R. DeCarlo, "Switched controller synthesis for the quadratic stabilisation of a pair of unstable linear systems," European Journal of Control, vol. 4, no. 2, pp. 140-147, 1998.

[9] J. P. Hespanha and A. S. Morse, "Stability of switched systems with average dwell-time," in Proceedings of the 38th IEEE Conference on Decision and Control (CDC '99), pp. 2655-2660, December 1999.

[10] G. Zhai, B. Hu, K. Yasuda, and A. N. Michel, “Disturbance attenuation properties of time-controlled switched systems," Journal of the Franklin Institute, vol. 338, no. 7, pp. 765-779, 2001.

[11] J. C. Geromel and P. Colaneri, "Stability and stabilization of continuous-time switched linear systems," SIAM Journal on Control and Optimization, vol. 45, no. 5, pp. 1915-1930, 2006.

[12] Z. R. Xiang and W. M. Xiang, "Stability analysis of switched systems under dynamical dwell time control approach," International Journal of Systems Science, vol. 40, no. 4, pp. 347-355, 2009.

[13] J. K. Hale and S. M. Verduyn Lunel, Introduction to Functional-Differential Equations, vol. 99 of Applied Mathematical Sciences, Springer, New York, NY, USA, 1993.

[14] D. Du, B. Jiang, P. Shi, and S. Zhou, " $H_{\infty}$ filtering of discrete-time switched systems with state delays via switched Lyapunov function approach," IEEE Transactions on Automatic Control, vol. 52, no. 8, pp. 1520-1525, 2007.

[15] S. Kim, S. A. Campbell, and X. Liu, "Stability of a class of linear switching systems with time delay," IEEE Transactions on Circuits and Systems. I. Regular Papers, vol. 53, no. 2, pp. 384-393, 2006.

[16] X.-M. Sun, J. Zhao, and D. J. Hill, "Stability and $L_{2}$-gain analysis for switched delay systems: a delaydependent method," Automatica, vol. 42, no. 10, pp. 1769-1774, 2006.

[17] W.-A. Zhang and L. Yu, "Stability analysis for discrete-time switched time-delay systems," Automatica, vol. 45, no. 10, pp. 2265-2271, 2009. 
[18] D. Xie, Q. Wang, and Y. Wu, “Average dwell-time approach to $L_{2}$ gain control synthesis of switched linear systems with time delay in detection of switching signal," IET Control Theory $\mathcal{E}$ Applications, vol. 3, no. 6, pp. 763-771, 2009.

[19] L. Wu, T. Qi, and Z. Feng, "Average dwell time approach to $L_{2}-L_{\infty}$ control of switched delay systems via dynamic output feedback," IET Control Theory \& Applications, vol. 3, no. 10, pp. 1425-1436, 2009.

[20] Y. G. Sun, L. Wang, and G. Xie, "Exponential stability of switched systems with interval time-varying delay," IET Control Theory \& Applications, vol. 3, no. 8, pp. 1033-1040, 2009.

[21] D. Wang, W. Wang, and P. Shi, "Exponential $H_{\infty}$ filtering for switched linear systems with interval time-varying delay," International Journal of Robust and Nonlinear Control, vol. 19, no. 5, pp. 532-551, 2009.

[22] D. Wang, W. Wang, and P. Shi, "Delay-dependent exponential stability for switched delay systems," Optimal Control Applications \& Methods, vol. 30, no. 4, pp. 383-397, 2009.

[23] Z. Xiang and Q. Chen, "Robust reliable stabilization of switched nonlinear systems with time-varying delays and delayed switching," Mathematical Problems in Engineering, vol. 2010, Article ID 471680, 2010.

[24] L. Dai, Singular Control Systems, vol. 118 of Lecture Notes in Control and Information Sciences, Springer, Berlin, Germany, 1989.

[25] G. Escobar, A. J. van der Schaft, and R. Ortega, "A Hamiltonian viewpoint in the modeling of switching power converters," Automatica, vol. 35, no. 3, pp. 445-452, 1999.

[26] B. Cantó, C. Coll, and E. Sánchez, "Positive N-periodic descriptor control systems," Systems E Control Letters, vol. 53, no. 5, pp. 407-414, 2004.

[27] B. Meng and J.-F. Zhang, "Output feedback based admissible control of switched linear singular systems," Acta Automatica Sinica, vol. 32, no. 2, pp. 179-185, 2006.

[28] J. Lin, S. Fei, and J. Shen, "Admissibility analysis and control synthesis for switched linear singular systems," Journal of Systems Engineering and Electronics, vol. 20, no. 5, pp. 1037-1044, 2009.

[29] G. Zhai, R. Kou, J. Imae, and T. Kobayashi, "Stability analysis and design for switched descriptor systems," International Journal of Control, Automation and Systems, vol. 7, no. 3, pp. 349-355, 2009.

[30] R. Shorten, M. Corless, K. Wulff, S. Klinge, and R. Middleton, "Quadratic stability and singular SISO switching systems," IEEE Transactions on Automatic Control, vol. 54, no. 11, pp. 2714-2718, 2009.

[31] B. Meng and J.-F. Zhang, "Reachability conditions for switched linear singular systems," IEEE Transactions on Automatic Control, vol. 51, no. 3, pp. 482-488, 2006.

[32] D. Koenig and B. Marx, " $H_{\infty}$-filtering and state feedback control for discrete-time switched descriptor systems," IET Control Theory \& Applications, vol. 3, no. 6, pp. 661-670, 2009.

[33] S. Ma, C. Zhang, and Z. Wu, "Delay-dependent stability of $H_{\infty}$ control for uncertain discrete switched singular systems with time-delay," Applied Mathematics and Computation, vol. 206, no. 1, pp. 413-424, 2008.

[34] T. C. Wang and Z. R. Gao, "Asymptotic stability criterion for a class of switched uncertain descriptor systems with time-delay," Acta Automatica Sinica, vol. 34, no. 8, pp. 1013-1016, 2008.

[35] E.-K. Boukas, Control of Singular Systems with Random Abrupt Changes, Communications and Control Engineering Series, Springer, Berlin, Germany, 2008.

[36] D. Zhaoping, Z. Qingling, and C. Guisong, "State feedback stabilization for switched singulå networked control systems with time-delay," in Proceedings of Chinese Control and Decision Conference (CCDC '09), pp. 5587-5591, June 2009.

[37] A. Haidar, E. K. Boukas, S. Xu, and J. Lam, "Exponential stability and static output feedback stabilisation of singular time-delay systems with saturating actuators," IET Control Theory $\mathcal{E}$ Applications, vol. 3, no. 9, pp. 1293-1305, 2009.

[38] V. Kharitonov, S. Mondié, and J. Collado, "Exponential estimates for neutral time-delay systems: an LMI approach," IEEE Transactions on Automatic Control, vol. 50, no. 5, pp. 666-670, 2005.

[39] S. Zhu, Z. Li, Z. Cheng, and J. Feng, "Delay-dependent robust stabilization for uncertain singular time-delay systems: Dynamic output feedback case," in Proceedings of the 44th IEEE Conference on Decision and Control, and the European Control Conference (CDC-ECC '05), pp. 5065-5070, December 2005.

[40] Q. Li, Q. Zhang, N. Yi, and Y. Yuan, "Robust passive control for uncertain time-delay singular systems," IEEE Transactions on Circuits and Systems. I. Regular Papers, vol. 56, no. 3, pp. 653-663, 2009.

[41] H. D. Tuan and P. Apkarian, "Low nonconvexity-rank bilinear matrix inequalities: algorithms and applications in robust controller and structure designs," IEEE Transactions on Automatic Control, vol. 45, no. 11, pp. 2111-2117, 2000. 


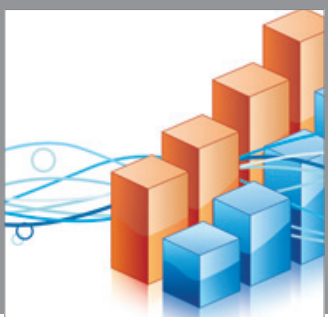

Advances in

Operations Research

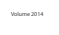

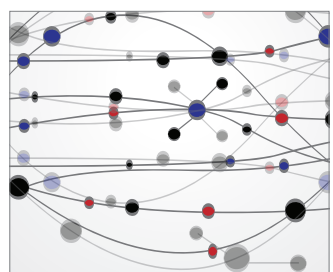

\section{The Scientific} World Journal
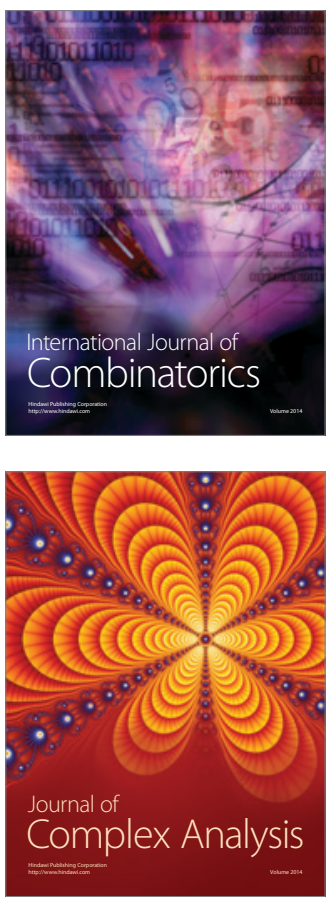

International Journal of

Mathematics and

Mathematical

Sciences
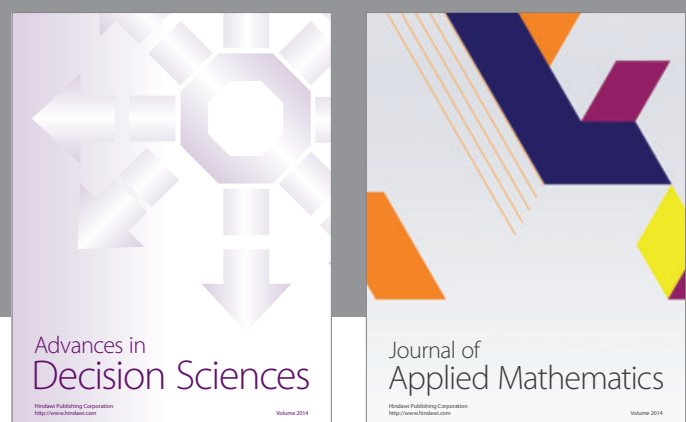

Journal of

Applied Mathematics
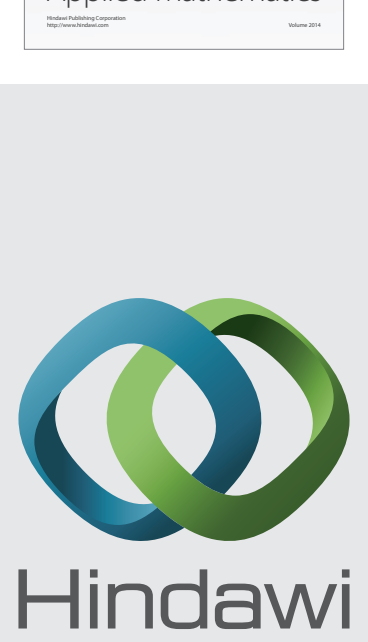

Submit your manuscripts at http://www.hindawi.com
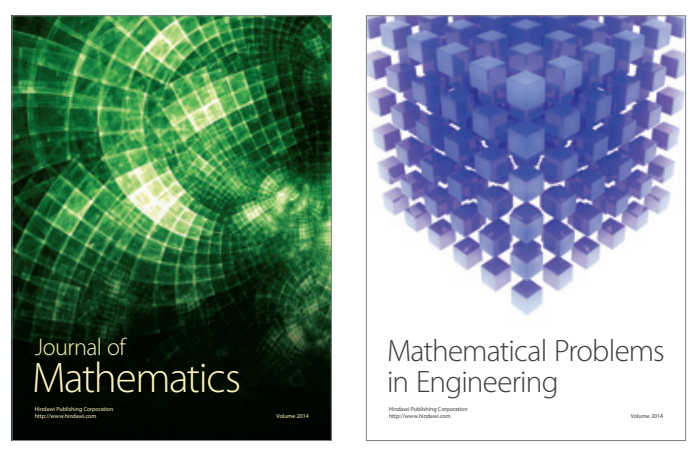

Mathematical Problems in Engineering
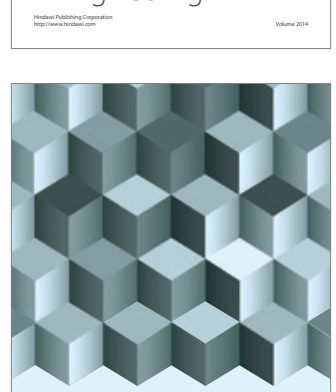

Journal of

Function Spaces
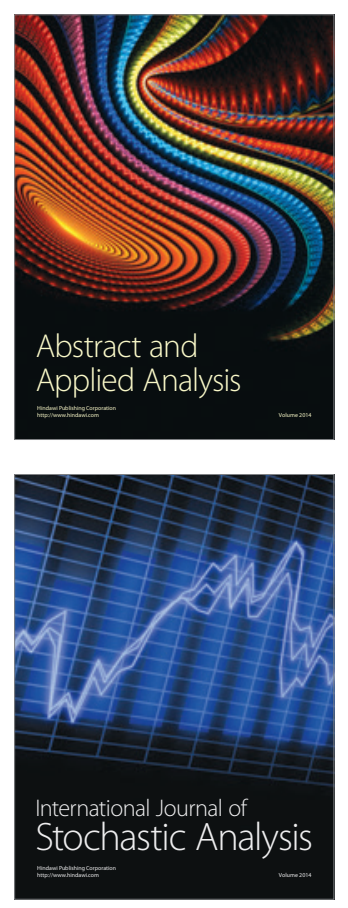

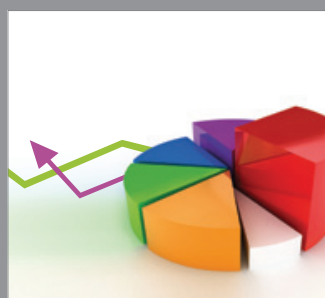

ournal of

Probability and Statistics

Promensencen
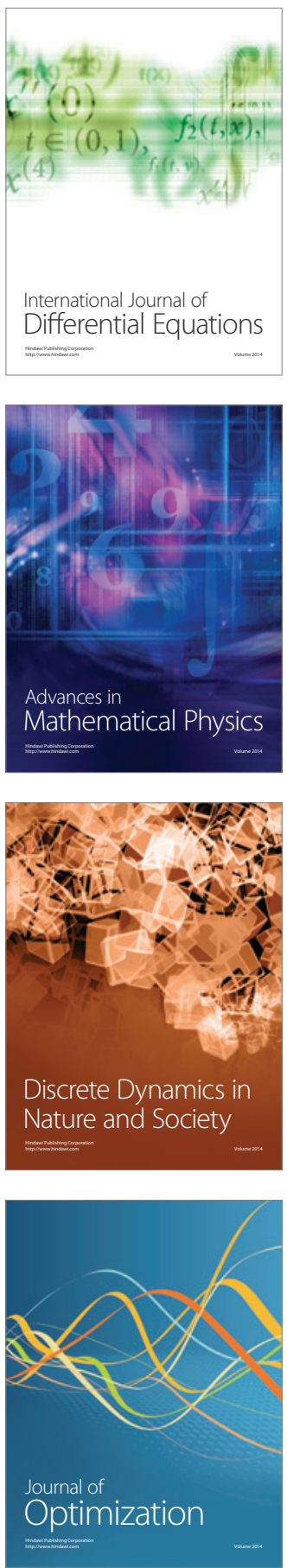